See discussions, stats, and author profiles for this publication at: https://www.researchgate.net/publication/327866877

\title{
Judgment Error in Lottery Play: When the Hot-Hand Meets the Gambler's
} Fallacy

Preprint $\cdot$ September 2018

CITATIONS

0

4 authors, including:

Q Qingxia Kong

Erasmus University Rotterdam

13 PUBLICATIONS 30 CITATIONS

SEE PROFILE
READS

77

(2.) Georg D. Granic

14 PUBLICATIONS 38 CITATIONS

SEE PROFILE 


\title{
Judgment Error in Lottery Play: When the Hot-Hand Meets the Gambler's Fallacy*
}

\author{
Qingxia Kong $^{\dagger} \quad$ Georg D. Granic ${ }^{\ddagger}$ Nicolas S. Lambert ${ }^{\S}$ \\ Chung-Piaw Teo
}

October 3, 2018

\begin{abstract}
We demonstrate that lottery markets can exhibit the "hot-hand" phenomenon, in which past winning numbers tend to receive a greater share of the bets in future draws, even though past and future events are independent. This finding is surprising, as works by Clotfelter and Cook (1993) and Terrell (1994) have previously documented the presence of an opposite effect - the "gambler's fallacy" - in the U.S. lottery market. The current literature also suggests that the gambler's fallacy prevails when random numbers are generated by mechanical devices, such as in lottery games (Ayton and Fisher 2004, Burns and Corpus 2004, Caruso et al. 2010). We use two sets of naturally occurring data to show that both the gambler's fallacy and the hot-hand fallacy can exist in different types of lottery games. We then run online experimental studies that mimic lottery games with one, two, or three winning numbers. Our experimental results show that the number of winning prizes impacts behavior. In particular, whereas a single-prize game leads to a strong presence of the gambler's fallacy, we observe a significant increase in hot-hand behavior in multiple-prize games with two or three winning numbers.
\end{abstract}

Keywords: Perception of Randomness, Hot-Hand Fallacy, Gambler's Fallacy, Lottery Game

*We thank Teck Ho, Stephen Leider, Thomas Peeters, Matthew Rabin, Peter Wakker, and Yaozhng Wu for helpful discussions. Granic gratefully acknowledges financial support from the Netherlands Organisation for Scientific Research through VIDI project 452-13-013. Lambert is grateful to the National University of Singapore for hosting him during part of this research, as well as the Yale University Cowles Foundation, and thanks the National Science Foundation for support under grant No. 1101209.

${ }^{\dagger}$ Rotterdam School of Management, Erasmus University; q.kong@rsm.nl.

${ }^{\ddagger}$ Erasmus School of Economics, Erasmus University; granic@ese.eur.nl.

$\S$ Graduate School of Business, Stanford University; nlambert@stanford.edu.

`Department of Decision Sciences, National University of Singapore; bizteocp@nus.edu.sg. 


\section{Introduction}

Studies show that people rely on heuristics or simple mental models to interpret random events (Oskarsson et al. 2009, Tversky and Kahneman 1974). Although such heuristics ensure fast decisions that generate reasonably good outcomes under most circumstances, they can trigger systematic deviations from certain notions of rationality. Among others, the gambler's and hot-hand fallacies are cognitive biases that have been commonly observed in the perception of randomness and they lead us to misjudge probabilities.

The gambler's fallacy is an erroneous belief in negative correlations of independent outcomes generated by a random process. For example, when tossing a fair coin, people often mistakenly believe that a tail is due after a sequence of heads. The gambler's fallacy has long been observed in the field, particularly in lottery games (e.g., Clotfelter and Cook 1993, Sundali and Croson 2006. Terrell 1994). The hot-hand fallacy - the counterpart to the gambler's fallacy - is the fallacious belief that a person who has experienced a success in one random event is more likely to succeed in subsequent attempts. This fallacy was first described by Gilovich et al. (1985) in their research on basketball games; they observed that both players and fans believe that a player who has made a successful streak of shots will continue to score as if having "hot-hands." Later, Camerer (1989) showed that betting markets for basketball games also exhibit a small hot-hand bias.

Although the two fallacies seem to contradict each other, the current literature shows that they may coexist in the same individual and/or appear within the same setting. For example, Croson and Sundali (2005) and Sundali and Croson (2006) found that casino players exhibited the gambler's fallacy when they bet on numbers, but the hot-hand fallacy regarding their own luck. Guryan and Kearney (2008) examined the sales data for lottery outlets that had sold winning jackpot tickets, and showed that those stores experienced a significant increase in game-specific ticket sales, exhibiting a "lucky store" effect.

The current literature posits two main explanations for the coexistence of these seemingly opposite phenomena. One line of research suggests that they both arise from the representativeness heuristics or belief in the law of small numbers (e.g., Asparouhova et al. 2009, Rabin 2002, Rabin and Vayanos 2010, Tversky and Kahneman 1971). These works identify the importance of streak length in influencing the perception of randomness: The gambler's fallacy prevails in short streaks, but as the streaks lengthen, beliefs in the hot-hand fallacy dominate. In the other line of research, observations that the gambler's fallacy is more prevalent in lottery games, and the hot-hand fallacy more prevalent in games that require skill, are often attributed to the source of the random number generator. That is, we tend to observe the gambler's fallacy if the random generator is a mechanical device and the hot-hand fallacy if it is a human being (e.g., Ayton and Fisher 2004, Burns and Corpus 2004, Caruso et al. 2010).

This paper provides empirical evidence using two sets of naturally occurring data to demon- 
strate that both the gambler's and the hot-hand fallacies can appear in lottery games. We collected field data from two fixed-odds lottery games. The first is a 3-digit (3D) lottery game played daily in China, the scheme of which resembles the Pick 3 lottery game reported by Clotfelter and Cook (1993); the other game is a 4-digit (4D) lottery game played in Southeast Asia that picks 23 winning numbers at a time, three times per week. Consistent with previous studies, we observe the gambler's fallacy in the 3D lottery game. In contrast to what the literature would predict, we were surprised to discover the hot-hand fallacy in the 4D game. Upon reflection, long streaks are unlikely to be observed in lottery games, and, therefore, theories building on streaks in sequences of random events cannot be applied directly to these environments. In addition, because the winning numbers for both the $3 \mathrm{D}$ and the $4 \mathrm{D}$ games are machine-generated, theories that focus on the source of randomness would predict the gambler's fallacy in both games.

To bridge this gap, our paper proposes an alternative explanation for our field observations: We suggest that the lottery game design can play a role in shaping the perception of randomness. Specifically, we show that the number of winning prizes has an impact on the occurrence of the two fallacies: Single-prize games can lead to the gambler's fallacy while multiple-prize games, under appropriate conditions, may result in the hot-hand fallacy. In addition to the number of winning prizes, the two lottery games differ in other aspects, such as frequency of draw and sample population. We, therefore, test our conjecture in an experimental study that allows us to both isolate the effect of the number of prizes and to control for all other differences.

In the experiment, participants are asked to guess the outcome of simulated lottery games that only differ with respect to the number of prizes. This design contributes to the experimental literature on the gambler's fallacy, which mainly focuses on binary choice sets and a single prize. We expand the choice set beyond the binary case and examine how behavior changes when multiple prizes are present. Our main results show that, whereas the gambler's fallacy still prevails in the single-prize game, the fallacy immediately disappears when the number of prizes increases to two or three. At the individual level, we observe more hot-hand behavior with multiple prizes than with a single prize. We infer from our observations that the game design, in the form of the number of prizes drawn, can systematically influence a player's betting behavior.

We further analyze our experimental data to understand why multiple-prize games lead to more hot-hand behavior. We hypothesize that different game experiences across single and multiple-prize games may play an important role. Specifically, single and multiple-prize games differ with respect to how frequently past winning numbers re-occur. In our experimental data, strong gambler's fallacy effects emerge in the first few rounds across all lottery games, suggesting that participants start out with a predisposition to avoid past winning numbers. This observation is consistent with cognitive evidence on the prevalence of the gambler's fallacy, 
Table 1: The probability of winning and seeing at least one repetition in subsequent draws

\begin{tabular}{lcc}
\hline & 3D game & 4D game \\
& $\begin{array}{c}1,000 \text { numbers } \\
\text { winning number }\end{array}$ & $\begin{array}{c}\text { 10,000 numbers } \\
23 \text { winning numbers }\end{array}$ \\
\hline $\begin{array}{l}\text { Probability of winning } \\
\begin{array}{l}\text { Probability of having at least one } \\
\text { repetition in two subsequent draws }\end{array}\end{array}$ & $\frac{1}{1000}=0.001$ & $1-\left(1-\frac{1}{10000}\right)^{23} \approx 0.0023$ \\
\hline
\end{tabular}

which is linked to the belief in negative autocorrelation (see Rabin (2002), Section 2, for a detailed literature review). Repetitions of winning numbers in two consecutive draws are rather rare events in our single-prize setup (10\% chance of repetition). The subjective game experience is therefore consistent with a belief in negative autocorrelation as contradicting evidence is rarely experienced. However, the probability of observing the repetitions of winning numbers in our multi-prize setups is much higher (37.7\% in the two-prize game and $70.8 \%$ in the threeprize game) and participants in multi-prize games frequently experience past winning numbers being re-drawn. We argue that, when participants are eventually confronted with frequent repetitions, they can abandon their initial belief in negative autocorrelation (gambler's fallacy), and adopt a new belief in positive autocorrelation (hot-hand fallacy), which is consistent with their subjective game experience. This interpretation is compatible with our observation that the gambler's fallacy quickly disappears after playing several rounds of multiple-prize games, whereas it persists in the single-prize game.

This interpretation can also explain the differences in our field data's behavior between the $3 \mathrm{D}$ and the 4D lottery games. The probabilities are calculated for two different games - one with 1 in 1,000 numbers winning (the 3D numbers game), and another with 23 in 10,000 numbers winning (the 4D numbers game). The second game, with 23 winners, clearly has a higher chance of seeing a winning number again in the next draw. If players observe that a winning number (or some related numbers) has a higher chance of appearing again, we ask: How will such observations affect their choice of lottery numbers to bet on? Our empirical data suggest that the gambler's fallacy prevails in the first case (1 prize in 1,000 numbers), whereas the hot-hand fallacy prevails in the latter (23 prizes in 10,000 numbers).

In summary, we provide empirical and experimental evidence that behavior can be altered through a careful lottery-system design. We believe these results can have important practical implications for risk management and decision making under uncertainty because they suggest that the perception of randomness can be manipulated, and hence, behavior can be nudged with the appropriate design. For instance, several countries have attempted to influence commuters' behavior by offering incentive schemes in which commuters earn credit for each journey taken (with triple credit for off-peak journeys) and to enter in weekly cash lotteries. The success of 
these schemes hinges on an insight from behavioral economics: On average, people are riskseeking when the stakes are small. Hence, a 1-in-1000 chance to win $\$ 100$ is more attractive than a cash award of $\$ 0.10$. For such low probability events, our results suggest that we could do better if we can induce the belief that certain choices made by players are more likely to win. For example, this could be done by designing lottery games that make the hot-hand fallacy dominate, and our results suggest that increasing the number of prizes is enough to make what is actually a 1-in-1,000 chance of winning appear to be a much more attractive bet to commuters.

\section{Literature Review}

Both the gambler's and the hot-hand fallacies have long been observed in laboratory experiments and in the field. Among many others, Clotfelter and Cook (1993) observed that lottery players in 3D numbers games in the United States are subject to the gambler's fallacy. Figure 1 shows the percentiles of betting ratios (betting volume index over average index on a particular day) on different days after the winning numbers have been drawn. Once a 3D number is drawn as the winner, subsequent betting ratios drop immediately and then gradually pick up. The immediate initial drop provides strong evidence of the gambler's fallacy at work. In a different context, Camerer (1989) showed that betting markets for basketball games exhibit a small hot-hand bias. Similarly, when examining a set of panel data, Green and Zwiebel (2017) found strong hot-hand fallacy effects among baseball players in Major League Baseball. Suetens et al. (2016) found that people playing Lotto games usually avoid numbers that have recently been drawn, exhibiting the gambler's fallacy, but tend to bet more on winning numbers in streaks (i.e., those that have been drawn several times in a row), suggesting the presence of the hothand fallacy. Chen et al. (2016) examined independent data sets from three empirical settings involving high-stake, sequential decisions: asylum judges, loan officers, and baseball umpires. They found evidence for negatively correlated decisions, consistent with the gambler's fallacy.

Importantly, both the gambler's and the hot-hand fallacies may coexist in the same individual and appear within the same setting. One line of research posits that they both arise from a representativeness heuristic or belief in the law of small numbers. Tversky and Kahneman (1971) coined this term and examined its connection with the gambler's fallacy. Rabin (2002) and Rabin and Vayanos (2010) built theoretical models to model the law of small numbers that directly leads to the gambler's fallacy. Asparouhova et al. (2009) ran a set of lab experiments on binary choice games, and their results support the work of Rabin (2002).

These studies built the decision biases (gambler's fallacy) directly into their models and showed that other decision biases may emerge as the streaks of winning lengthen. However, as streaks are unlikely in lottery games, these models cannot be directly applied in these 


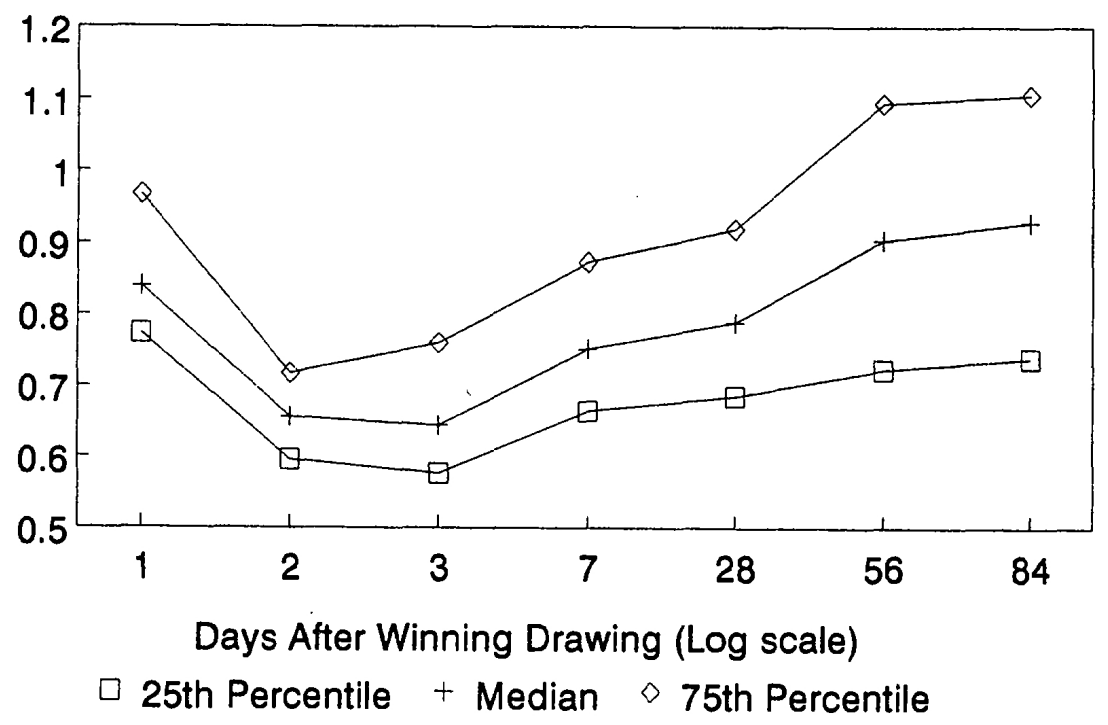

Figure 1: Betting Ratios on Previous Winning Numbers in 3-Digit Numbers Games (Clotfelter and Cook 1993)

environments. Our paper instead examines the role of lottery game design in shaping the perception of randomness. We show that, due to game design, either the gambler's or the hothand fallacy can arise under appropriate conditions, and we can thus manipulate the behaviors through careful system design.

A second stream of research argues that the type of random-event generator - whether an inanimate device or a human being - determines the occurrence of the gambler's or hot-hand fallacy (Ayton and Fisher 2004, Burns and Corpus 2004). Specifically, if the random process is believed to be generated by a mechanical device, people expect negative recency; if a human being generates the sequences, however, people expect positive recency. For example, the experimental study by Caruso et al. (2010) demonstrated that human subjects tend to predict the continuation of a streak of outcomes when the agents that generate it are perceived to be intentional. In contrast, when generated by a mechanical device (as in lottery games), people believe the streak will end; that is, the gambler's fallacy dominates. These results are reinforced by field observations made in casinos and lottery stores (Sundali and Croson 2006. Guryan and Kearney 2008). Green et al. (2010) developed several ingenious arguments to demonstrate that experimental cues can alter how subjects learn about the generating process (using optimal Bayesian updating with erroneous beliefs) and thus substantially affect subjects' choices, without altering the underlying outcome probabilities. Our work complements these explanations; our empirical evidence shows that either the gambler's or the hot-hand fallacy can occur even if the random process is generated by mechanical devices.

In addition, further evidence of cognitive biases also suggests that the gambler's fallacy 
occurs in situations in which information is experienced sequentially over time (Baron and Leider 2010). For instance, Militana et al. (2010) found that the bias was stronger when research subjects had longer time intervals between choices. In contrast, the gambler's fallacy decreased when people's inaccurate assessments of the probability of an outcome imposed costs (Terrell 1994).

Navarrete and Santamari (2012) showed, in two lab experiments, that the incidence of the gambler's fallacy was reduced when the size of the choice set was increased. Our paper has a different focus: We investigate how the number of prizes - the size of the outcome set-influences player's betting behavior while fixing the size of the choice set. Our results, consequently, are different: We observe more hot-hand behavior in multiple-prize games compared to single-prize games.

\section{$3 \quad$ Field Evidence}

In this section, we use field data from two fixed-odds lottery games to demonstrate that both the gambler's fallacy and the hot-hand fallacy can exist in lottery games. The first is a 3-digit (3D) lottery game played in China, the scheme of which resembles the "Pick 3" lottery game reported in Clotfelter and Cook (1993). The lottery draws a single prize-winning number every day, seven days a week. The other game, a 4-digit (4D) lottery game played in Southeast Asia, picks 23 prize-winning numbers at a time, three times a week. In what follows, we describe the two games in detail and provide evidence that the gambler's fallacy prevails in the 3D game while the hot-hand fallacy is the dominant behavior in the $4 \mathrm{D}$ game 1

\subsection{D Numbers Game}

In the 3D numbers game, a single winning number from 000 to 999 is randomly drawn with equal probability at 8:30 p.m. each day. Lottery tickets for the day's draw are sold on the same day until 8:00 p.m. Each wager costs 2 RMB. There are three types of bets: Straight, Box 3, and Box 6. In the Straight bet, players bet on a specific 3D number and receive 1,000 RMB per wager if the number wins. The Box 3 bet allows players to make one wager on the permutation of any 3D number with two identical digits, and in the Box 6 bet, on the permutation of any $3 \mathrm{D}$ number with three unique digits. For example, a player who places a 2 RMB wager on 223 and chooses the Box 3 bet type actually bets 2/3 RMB on each of the 3 numbers 223, 322, and 232. The payout per wager ${ }^{2}$ and odds of winning are shown in Table 2.

\footnotetext{
${ }^{1}$ We ran statistical tests for each lottery game and found no evidence of lag-one autocorrelation among the winning numbers. The details are in Appendix A.

${ }^{2}$ There are minor payout variations by areas: In Beijing, the game pays 980 per wager for the Straight bet, and in Shanghai and Tibet, the payout is 333 for the Box 3 bet and 166 for the Box 6 bet.
} 
Table 2: Prize Structure and Winning Odds in 3D Numbers Game

\begin{tabular}{lccc}
\hline Bet Type & Match to Win & Payout per Wager & Odds \\
\hline Straight & Match the exact order & 1,000 & 1 in 1,000 \\
Box 3 & Match any order (2 identical digits) & 320 & 1 in 333 \\
Box 6 & Match any order (3 unique digits) & 160 & 1 in 167 \\
\hline
\end{tabular}

Testing for the presence of the gambler's fallacy usually requires a full dataset that includes the sales of every number in every draw. Such a dataset is not publicly available for the lottery of interest. Nevertheless, we were able to obtain a rich enough dataset from the official website of the game operator ${ }^{3}$, which consists of (i) the winning number drawn; (ii) the number of winning wagers (of the three types), and (iii) total sales for each draw. We extract this information from 4,466 draws on a daily basis over a 150-month period-from May 8, 2005, to October 30, 2017.

In this dataset, 10 numbers were never drawn as prize winners, 59 numbers appeared only once, and 931 numbers were drawn at least twice. We define our dependent variable, "payout rate," as the amount paid to bettors relative to total sales for a given draw. The higher the payout rate of a winning number the more popular this number was among bettors relative to the market size of the draw. The overall mean payout rate is $49.2 \%$ and the median is $42.7 \%$. The lowest payout rate of $7.3 \%$ occurred on September 18, 2010, when "945" was drawn as the winning number; the highest payout rate was $530.3 \%$, which occurred on July 18, 2005, when "353" was drawn as the winning number. A Welch's two sample t-test does not detect any significant difference $(p$-value $=0.329)$ in mean payout rates between numbers that have been drawn only once (with mean $m=0.526$ ) and numbers that have been drawn at least twice $(m=0.488)$.

We seek to estimate the change in subsequent sales of a 3D number after it has been drawn as the winning number. As we only have sales information for winning numbers, we construct a subsample of 3,476 draws in which the winning numbers have been drawn previously (repeated) at least once within the period the full dataset covers. To measure the influence of past wins on future ticket sales, we define the "Delay" of a winning number as the number of draws that have elapsed since last time it was drawn. We then bin the draws according to the Delay, with intervals of a week. Each bin contains around 30 data points (draws). Figure 2a shows the 25 th percentile, the median, and the 75 th percentile of the payout rates in each bin for the first eight weeks. The first data point represents the same statistics for the full dataset. We observe a clear pattern of declining payout rates with a one-week Delay, which then eventually bounce back to their initial levels. Bettors display a tendency to avoid past winning numbers with

\footnotetext{
${ }^{3}$ http://www .zhcw.com/3d/kaijiangshuju/index . shtml.
} 
short delays, exhibiting strong evidence for the gambler's fallacy. A more detailed account on the data underlying Figure 2a is provided in Table 9 in Appendix B.

Our latter interpretation of the data is corroborated by a series of paired t-tests. For each number, we calculate its average payout rate for all draws that repeat within $x$ weeks (early repetition) and that repeat in more than $x$ weeks (late repetition). We compare average payout rates between "early" and "late" repetitions at the intra-number level. We then test the null hypothesis of equal payout rates between early and late repetitions for varying values of $x$, with $x=3,5,7,9$, and 11 . All tests unanimously show that the mean payout rate for early repetitions is significantly lower than that for late repetitions, and it is worth noting that all p-values are in the order of magnitude of $10^{-5}$ (see detailed results in Table 10 in Appendix B). However, one drawback of our approach is that we must exclude numbers that lack either early or late repetition. As a next step, we will demonstrate the existence of the gambler's fallacy in $3 \mathrm{D}$ games via a regression analysis that takes into account a broader set of winning numbers.

Figure 2: Betting on Previous Winning Numbers in 3D and 4D

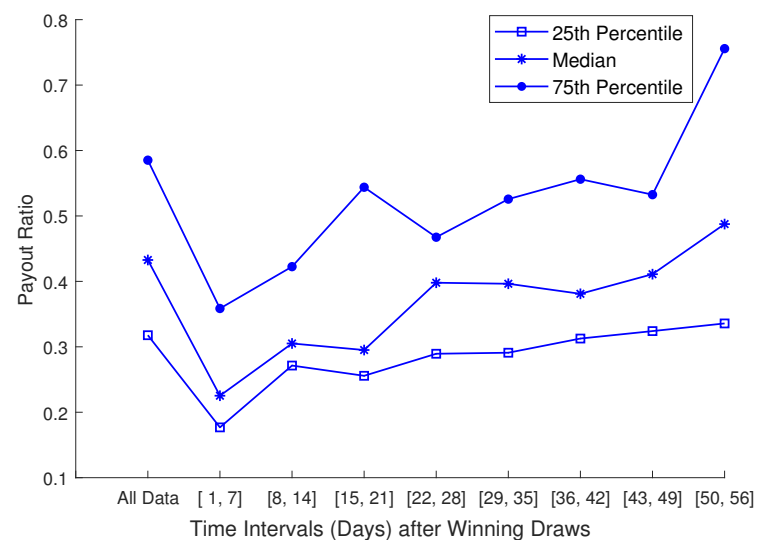

Figure 2a: 3D Game

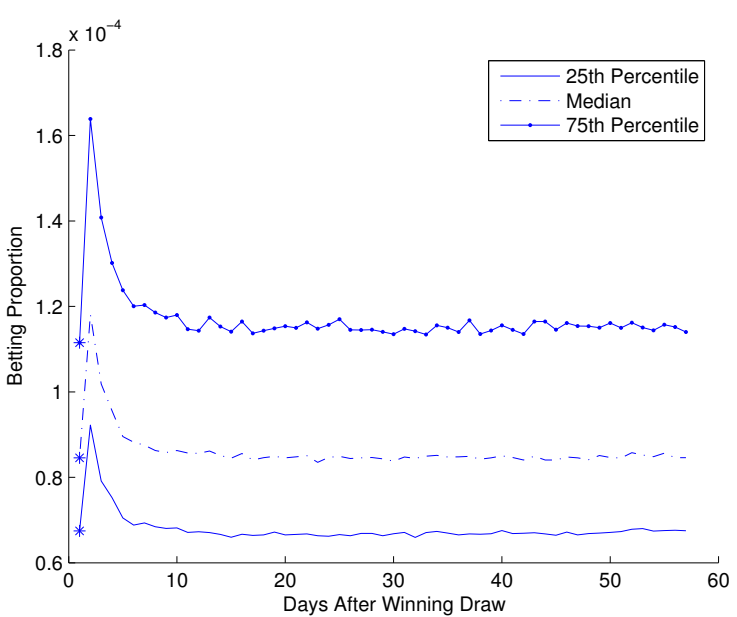

Figure 2b: 4D Game

For the regression analysis, we again consider all numbers that were drawn at least twice, this time including the first draw so that we can estimate the payout rates at 0 for the horizontal axis. We use the demeaned payout rate, denoted as $D R$, as the dependent variable. To obtain the demeaned payout rates, we first calculate the average payout rate for each number in all draws of the sample, then subtract this number-specific average from the observed payout rate of the winning number in each draw. Demeaning mimics a number fixed-effects approach, and allows us to control for number-specific effects (e.g., superstitions, lucky numbers, etc.) 迎 For each

\footnotetext{
${ }^{4}$ Since winning numbers are drawn identically and independently, demeaning and a fixed-effects approach should theoretically yield the same estimated effect of past wins on future betting behavior. We decided not
} 
Table 3: OLS Estimated Regression Coefficients on Payout Ratio in 3D \& 4D Numbers Games

\begin{tabular}{lcc}
\hline & $3 \mathrm{D}$ & $4 \mathrm{D}$ \\
\hline $\log _{10}(T)$ & $-1.817^{* * *}$ & $0.233^{* * *}$ \\
$\left(\log _{10}(T)\right)^{2}$ & $3.683^{* * *}$ & $-0.286^{* * *}$ \\
& $(0.954)$ & $(0.100)$ \\
$\left(\log _{10}(T)\right)^{3}$ & $-2.969^{* * *}$ & $0.086^{* * *}$ \\
& $(0.942)$ & $(0.032)$ \\
$\left(\log _{10}(T)\right)^{4}$ & $1.181^{* * *}$ & \\
& $(0.446)$ & \\
$\left(\log _{10}(T)\right)^{5}$ & $-0.231^{* *}$ & \\
& $(0.101)$ & \\
$\left(\log _{10}(T)\right)^{6}$ & $0.018^{* *}$ & \\
& $(0.009)$ & \\
Intercept & $0.014^{*}$ & -0.005 \\
& $(0.009)$ & $(0.006)$ \\
\hline Observations & 4,407 & 1,103 \\
F-value & 7.03 & 3.06 \\
degrees of freedom F-value & $(6,4400)$ & $(3,1099)$ \\
Robust std. err. & $\mathrm{Y}$ & $\mathrm{Y}$ \\
\hline
\end{tabular}

Notes: Standard errors are robust and provided in parentheses.

Significance Coding: *** - 1\%,** - 5\%,* $-10 \%$.

number in the sample, we calculate the average payout ratio and subtract this number-specific average from the actual observed payout ratios for this number. By construction, the mean of the demeaned payout ratio is zero. We use the Delay (denoted as $T$ ) as the independent variable. We characterize the relationship between $D R$ and $\log _{10}(T+1)$ as a higher-degree polynomial. Polynomials are functionally flexible, and their curvatures can capture the type of effects we were expecting to observe in the data: a diminishing effect over time of past wins on current payout rates. We use an ordinary least squares approach with robust standard errors to estimate the effect of $\log _{10}(T+1)$ on $D R$. We start with the quadratic function and add successively higher-degree polynomial terms, as long as they turn out to be significant. Our final model for the 3D game is a 6th degree polynomial, and takes the following form:

$$
D R_{i}=\beta_{0}+\sum_{k=1}^{6} \beta_{k} \times\left(\log _{10}\left(T_{i}+1\right)\right)^{k}+\alpha_{i}
$$

in which $D R_{i}$ is the demeaned payout ratio of the $i-t h$ draw in the subsample; $\beta_{0}$ is the fixed intercept, $\beta_{k}$ is the coefficient of the $k^{\text {th }}$ order polynomial function, and $\alpha_{i} \sim N\left(0, \sigma_{\alpha}^{2}\right)$ to follow the fixed effects approach, however, as this would have required us to estimate an additional 990 parameters in a model with 4,407 total observations. 
represents unobserved random effects. Estimated model coefficients are shown in Table 3. All estimated parameters are significant with small $p$-values.

To visualize our estimation results, we plot the predicted values and their $95 \%$ confidence interval in Figure 3a. We observe a strong gambler's fallacy effect, in which the demeaned payout rate significantly drops after a winning draw, gradually picks up, and goes back to its intial level after around 60 draws. At a delay of one day, payout rates are reduced by almost 30 percentage points. Even at a delay of 10 days, payout rates are down by 10 percentage points.

Figure 3: Estimated Average Payout Rate and 95\% CI of Previous Winning Numbers in 3D and 4D after a Winning Draw

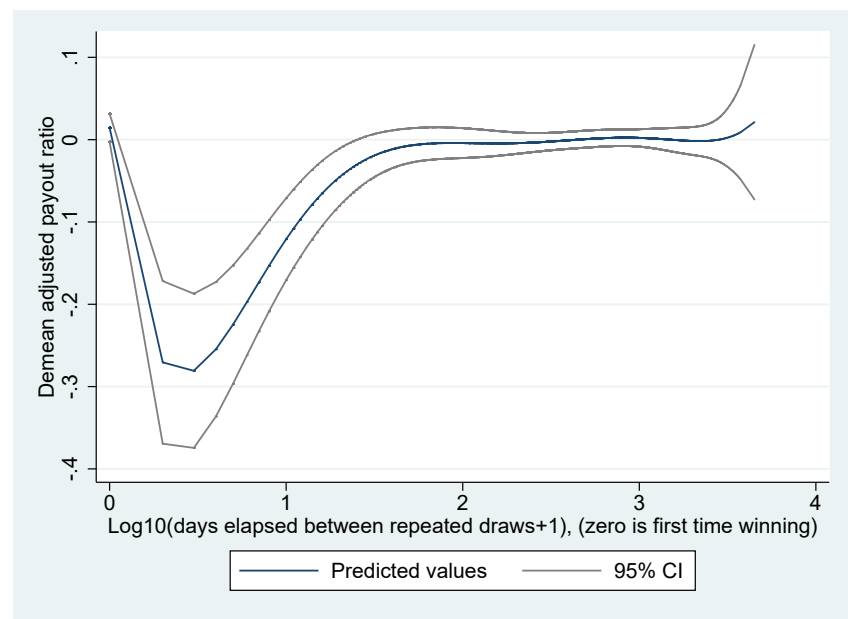

Figure 3a: 3D Game

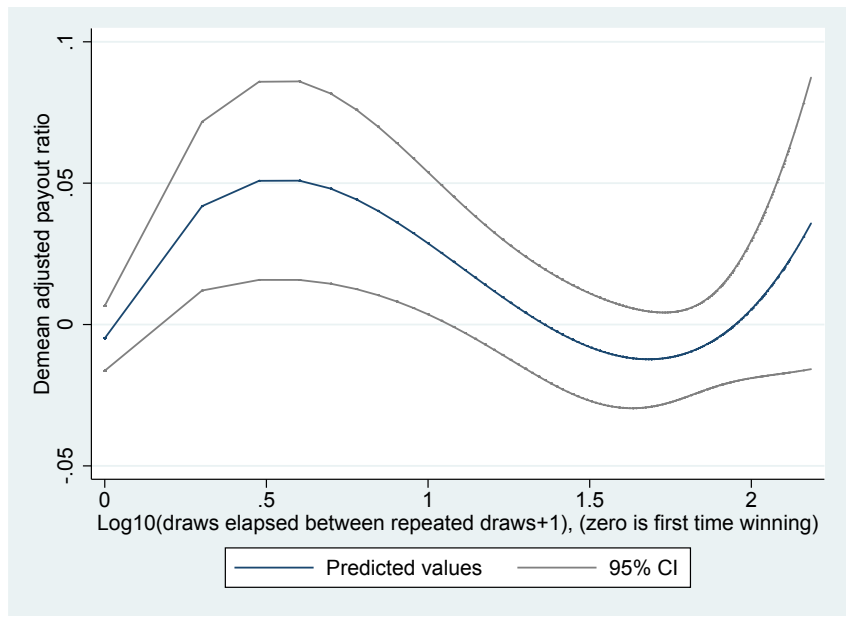

Figure 3b: 4D Game

\subsection{D Numbers Game}

In the $4 \mathrm{D}$ lottery game, players bet on numbers 0 to 9,999 . In contrast to the 3D game, in which sales only occur on the day of the draw, 4D sales start one week before each draw and close at 6 p.m. on the day of the draw. There are three draws every week, on Wednesday, Saturday, and Sunday. The minimum cost of a bet is one local currency. At each draw, 23 numbers are picked as winning numbers, randomly generated with replacement. There are five prize categories. The first, second, and third prize categories correspond to the first, second, and third winning number drawn, and offer a per-wager payout of 2,000, 1,000, and 500 local currency, respectively. The next ten numbers belong to the so-called "Starters" category, with a payout of 250 per wager. Lastly, the remaining 10 numbers belong to the "Consolation" category, with a payout of 60 per wager. Table 4 shows the payout for each prize category and its winning odds. 
Table 4: Prize Table and Winning Odds in 4D Numbers Games

\begin{tabular}{ccc}
\hline Prize Category & Payout per Wager & Odds \\
\hline 1st Prize & 2,000 & 1 in 10,000 \\
2nd Prize & 1,000 & 1 in 10,000 \\
3rd Prize & 500 & 1 in 10,000 \\
10 Starter & 250 & 1 in 1,000 \\
10 Consolation & 60 & 1 in 1,000 \\
\hline
\end{tabular}

We investigate the effect of a winning draw on subsequent betting behavior for the winning numbers. We aim to demonstrate that a simple change in the game design - from drawing one winning ball in 3D to drawing 23 winning balls in 4D - substantially impacts betting behavior. Whereas we have established the gambler's fallacy as the prevalent behavior in the 3D game, we will now establish that hot-hand behavior is prevalent in the $4 \mathrm{D}$ game.

We obtained data from a game operator in the Southeast Asia region, with information on 156 draws over a 1-year period. The dataset consists of sales volume for each of the 10,000 $4 \mathrm{D}$ numbers and the 23 winning numbers in each draw. Due to the presence of different prize categories in the $4 \mathrm{D}$ numbers game, we use the betting proportion - defined as the sales volume on a number divided by total sales in a particular draw - as our measure for the popularity of a number $5^{5}$ We examine all winning numbers from draw 1 to draw 100 and calculate their betting proportions, respectively, on the day they are drawn and the subsequent 56 draws. Sales increase by about $40 \%$ immediately following a winning draw (cf. Figure $2 \mathrm{~b}$ ), suggesting that the hot-hand fallacy prevails at the aggregate level. Next, we will demonstrate the existence of the hot-hand fallacy in $4 \mathrm{D}$ via rigorous regression analysis.

To facilitate a fair comparison with the 3D game, we also construct a subsample of 1,103 draws, consisting of winning numbers that have been drawn at least twice in our dataset. We denote $D R$ as the demeaned value of the betting proportion and $T$ as the number of draws elapsed since this winning number last won. We characterize the relationship between $D R$ and $\log _{10}(T+1)$ as a higher-degree polynomial. The final relationship after adding significant higher-order polynomial terms is a 3rd-order polynomial, and takes the following form:

$$
D R_{i}=\beta_{0}+\sum_{k=1}^{3} \beta_{k} \times\left(\log _{10}\left(T_{i}+1\right)\right)^{k}+\alpha_{i}
$$

Corresponding regression results are reported in Table 3. All polynomials coefficients are significant, and the signs are opposite to those of corresponding results in $3 \mathrm{D}$. To visualize our

\footnotetext{
${ }^{5}$ The change in the dependent variable from payout rates in $3 \mathrm{D}$ to betting proportions in $4 \mathrm{D}$ is innocuous. The payout rate in the 3D game is a simple linear function of betting proportion. All of our 3D results hold true if we replace payout rates with betting proportions.
} 
estimated effects, we plot the predicted values and their $95 \%$ confidence interval in Figure $3 \mathrm{~b}$. We observe an increase in the demeaned betting proportion for short Delays, which is a clear manifestation of bettors actively seeking past winning numbers. This effect gradually decreases as the Delay becomes longer, with betting proportions reaching their initial levels at around 25 winning draws. This result suggests the existence of the hot-hand fallacy in the 4D numbers games.

In summary, we observe that lottery players' betting behaviors are significantly affected by the gambler's fallacy in the 3D game and the hot-hand fallacy in the 4D game. We conjecture that a plausible explanation is that the game design - in particular, the size of outcome sets - leads to the different observations in the two fixed-odds lottery games. In the 4D game there are 23 winning numbers in each draw; repetition of winning numbers across draws therefore occurs more frequently and similarities across draws can be easily observed. This observable repetition fosters the bettors' belief that the same or similar numbers have a higher chance of being drawn again, leading them to select past winning numbers. On the other hand, consecutive wins or extremely short delays in repetitions are rather rare events in the $3 \mathrm{D}$ game. From the bettors' perspective, it is therefore the norm not to observe recurring past winning numbers. This, in turn, can lead bettors to falsely infer that past wins have a lower chance of being drawn again, which motivates them to avoid past winning numbers.

Nevertheless, we cannot rule out other factors that might be the driving force behind our observations. For example, cultural differences might exist between the two populations. The two games also differ in the number of winning prizes, number of digits in the outcome set, and the frequency with which draws are staged. Due to data limitations, we cannot exclude the possibility that the observed differences between the games are the result of these structural differences. We therefore decided to examine how the game design influences betting behavior via a controlled experimental study. Our goal is to make the causal statement that a ceteris paribus change in the size of the winning numbers set increases bettors' tendency to seek past winning numbers, thereby confirming our conjecture about the mechanism underlying our empirical observations. In the following section, we report on the details of our experiment.

\section{Experimental Studies}

Our experimental study investigates how game design - in particular, the size of the set of winning numbers - affects gambler's and hot-hand fallacy behaviors via a simulated lottery game. We have developed an online simulated lottery game that allows us to induce ceteris paribus changes in the number of winning prizes drawn. This environment presents several advantages for investigating the mechanisms of human probabilistic predictions. The game 
is widespread in the population, requires little skill, and each draw from the lottery machine "follows a known, truly random process with a fixed probability for each number" (Suetens et al. 2016). In our game, a computer randomly draws winning number(s) from an urn containing 10 balls numbered from 0 to 9 . Each number is represented by exactly one ball in the urn, and each ball has an equal chance of being drawn ${ }^{6}$ Following Harrison and List (2004), our experiment can be classified as a framed field experiment. We refer the reader to Al-Ubaydli and List (2015) for a discussion of various parameters estimated by different types of experiments.

\subsection{Method}

\section{Participants}

We recruited 210 participants via the crowd-sourcing platform Prolific for our online experiment.7 Participants were screened based on English as first language to avoid any linguistic ambiguity for the instructional aspects of the experiment. Our participants were either U.K.or U.S.-based, comprising $89 \%$ and $8 \%$ of the sample, respectively; $58.6 \%$ were female, $12 \%$ were students, and the average age was 36.5 years. Self-reported median household income was between $\$ 35,000$ and $\$ 49,999$, and $66 \%$ of the participants reported that they played lottery or similar betting games less than once a month, with 35\% stating that they never played such games at all. On average, it took participants eight minutes to complete the experiment. They received a flat completion fee of $£ 0.80$ (approx. \$1.05). The best-guessing participant within a treatment was awarded a bonus payment of $£ 15$ (\$19.80). The expected remuneration per participant amounted to 1.5 times the recommended remuneration set by Prolific.

Design, apparatus, and procedure

Participants received an invitation link to the simulated lottery game via Prolifc. The lottery game itself was administered via Qualtrics, an online survey tool that recorded participants' self-paced responses. Screenshots of the decision screens as well as transcripts of the online instructions are provided in Appendix C. In total, there were 45 rounds with one draw of winning number(s) in each round (multiple winning numbers were drawn in one draw without replacement). The first five rounds constituted test rounds and gave participants the opportunity to familiarize themselves with the presentation style. In each of the remaining 40 rounds,

\footnotetext{
${ }^{6}$ Our choice for 10 balls is motivated by practical concerns. Our aim is to create an environment in which winning is less likely than losing, but still occurs frequently enough to make incentives salient. At the same time, we want enough flexibility to create different experiences with regard to how frequently past winning number reoccur. Ten has the additional advantage that it allows us to create a natural and homogeneous outcome set with regard to the number of digits present.

${ }^{7}$ Prolific is a U.K.-based crowd-sourcing platform that connects researchers with participants for their studies. In contrast to alternative platforms, such as Amazon's MTurk, Prolific is specifically geared toward the needs of researchers; participating in research studies is the only activity in which platform members can engage. The platform has been shown to produce reliable, high-quality data (cf. Peer et al. 2017).
} 
participants were asked to guess one number from 0 to 9 . If the guess matched either of the winning numbers drawn, the participant earned one point. Points were accumulated over the 40 guessing rounds, and the final score determined the winner of the prize. We mimicked the design of lottery websites as observed in 3D and 4D games. In particular, participants always saw the winning number(s) from the last round on screen and they could click a button to reveal the results of the five most recent rounds.

Participants were randomly assigned to three experimental conditions, which differed only with respect to how many winning numbers were drawn in each round. In treatment $T=1,75$ participants played the lottery game with one winning number drawn. In treatments $T=2$ and $T=3,63$ and 72 participants played lottery games with two and three winning numbers drawn without replacement, respectively ${ }^{8}$. We implemented a stratified randomization procedure, which explains the minimal differences in terms of number of participants across games.

\subsection{Results}

For our analysis of the 40 guessing rounds, we follow the same empirical strategy used by Suetens et al. (2016) and Wang et al. (2016), who analyze naturally occurring data with the same information structure as ours. We run probit regressions to estimate the effect of past winning draws on current betting behavior. Independent observations are taken at the participantperiod-number level. Our dependent variable takes the value one if a participant bets in a given period on a given number, and zero otherwise. Independent variables include a dummy for the most recent history (period $t-1$ ) and a count variable for number of wins in earlier histories (periods $t-5$ to $t-2$ ). For brevity of exposition, we only report estimation results for these two variables. However, all estimated models include fixed effects (FE) at the round and number level.

We also control for subject-specific "hotness" of lucky numbers. For this, we include a variable that measures the share of bets a number has received in the past. To control for other subject-specific effects, we either include subject FE or a set of demographic controls in terms of gender, age, income, and gambling habits. Standard errors are clustered at the participant level. Table 5 reports the average marginal effects of our regressions.

We will focus the discussion of our results on the model with subject FE, since differences across estimation models are minimal. We find that, if a number has won the most recent draw (period $t-1$ ), the probability of participants' betting on this particular number is reduced by 4.3 percentage points in $T=1$. This effect lasts beyond the most recent draw. For every win in the periods $t-2$ to $t-5$, the betting proportion on that number is reduced by 2.1

\footnotetext{
${ }^{8}$ Although the winning numbers in the $4 \mathrm{D}$ game are drawn with replacement, we do not observe any repetition in the winning numbers in our dataset. To avoid confusion among participants, we draw the winning numbers without replacement in the experiment, which was common knowledge.
} 
Table 5: Average Marginal Effects of Probit Regression on Historical Wins (Aggregate Level)

\begin{tabular}{lcccccc}
\hline & \multicolumn{2}{c}{$\mathrm{T}=1$} & \multicolumn{2}{c}{$\mathrm{T}=2$} & \multicolumn{2}{c}{$\mathrm{T}=3$} \\
\hline Won period t-1=1 & $-0.043^{* * *}$ & $-0.044^{* * *}$ & 0.004 & 0.008 & -0.011 & -0.009 \\
& $(0.007)$ & $(0.006)$ & $(0.014)$ & $(0.014)$ & $(0.009)$ & $(0.009)$ \\
Wins in t-2 to t-5 & $-0.021^{* * *}$ & $-0.017^{* * *}$ & -0.002 & 0.002 & -0.005 & -0.003 \\
& $(0.005)$ & $(0.005)$ & $(0.004)$ & $(0.003)$ & $(0.003)$ & $(0.003)$ \\
\hline Number of participants & 75 & 75 & 63 & 63 & 72 & 72 \\
Subject FE & $\mathrm{Y}$ & $\mathrm{N}$ & $\mathrm{Y}$ & $\mathrm{N}$ & $\mathrm{Y}$ & $\mathrm{N}$ \\
Period FE & $\mathrm{Y}$ & $\mathrm{Y}$ & $\mathrm{Y}$ & $\mathrm{Y}$ & $\mathrm{Y}$ & $\mathrm{Y}$ \\
Number FE & $\mathrm{Y}$ & $\mathrm{Y}$ & $\mathrm{Y}$ & $\mathrm{Y}$ & $\mathrm{Y}$ & $\mathrm{Y}$ \\
Demographic controls & $\mathrm{N}$ & $\mathrm{Y}$ & $\mathrm{N}$ & $\mathrm{Y}$ & $\mathrm{N}$ & $\mathrm{Y}$ \\
Clustered std. err. & $\mathrm{Y}$ & $\mathrm{Y}$ & $\mathrm{Y}$ & $\mathrm{Y}$ & $\mathrm{Y}$ & $\mathrm{Y}$ \\
Obs & 30000 & 30000 & 25200 & 25200 & 28800 & 28800 \\
\hline
\end{tabular}

Notes: Cluster robust standard errors are provided in parentheses. Significance Coding: *** $1 \%, * *-5 \%, *-10 \%$.

percentage points in $T=1$. On the aggregate level, we observe a strong gambler's fallacy effect in $T=1$. However, aggregate betting behavior is significantly impacted by a change in the number of winning balls drawn. In treatments $T=2$ and $T=3$, we do not detect any significant manifestation of the gambler's fallacy in aggregate betting behavior.

Next, we analyze participants' betting behavior conditional on streak length. When a number is drawn as a winner in consecutive rounds, we say that there is a "streak" of that winning number. The streak length is then defined as the number of consecutive winning rounds. For example, a streak length of one means that a number won in the most recent round $(t-1)$ but not in $(t-2)$, whereas a streak length of four means that a number won in round $(t-4)$ to $(t-1)$, but not in $(t-5)$. As discussed by Rabin (2002) and Rabin and Vayanos (2010), streak length plays a prominent role in the misperceptions of positive or negative autocorrelation in i.i.d. processes. We run probit regressions to estimate the impact of streak length on the betting proportion of a number, following the same empirical strategy we used in Table 5. Table 6 reports the corresponding average marginal effects. The absolute frequencies of streak-length occurrences per participant are underlined and provided above the coefficient estimates (e.g. 36 streaks of length one in $T=1,28$ streaks of length two in $T=3$ ). In $T=1$, we find the gambler's fallacy to emerge in short streaks (with streak lengths one and two), but then betting behavior switches and turns into hot-hand fallacy behavior when the streak length is four. It is worth noting that this pattern of behavior is compatible with the theoretical predictions derived by Asparouhova et al. (2009). In $T=2$, we find only hot-hand behavior with longer streaks. This behavior has already emerged when streak length is three and persists when streak length is four. In $T=3$, there is no significant impact of streak length on betting behavior. 
Table 6: Average Marginal Effects of Probit Regression on Streak Length (Aggregate Level)

\begin{tabular}{ccccccc}
\hline & \multicolumn{2}{c}{$\mathrm{T}=1$} & \multicolumn{2}{c}{$\mathrm{T}=2$} & \multicolumn{2}{c}{$\mathrm{T}=3$} \\
\hline Length streak $=1$ & \multicolumn{2}{c}{36} & $\frac{66}{6}$ & $\frac{85}{8}$ & \\
& $-0.051^{* * *}$ & $-0.053^{* * *}$ & -0.004 & 0.000 & -0.014 & -0.012 \\
& $(0.007)$ & $(0.006)$ & $(0.013)$ & $(0.013)$ & $(0.009)$ & $(0.009)$
\end{tabular}

Length streak $=2$

$$
\begin{array}{cccccc}
\frac{2}{2} & \multicolumn{2}{c}{10} & \multicolumn{2}{c}{28} \\
\left(0.054^{* * *}\right. & -0.044^{* *} & 0.013 & 0.018 & -0.002 & -0.000 \\
& (0.019) & (0.020) & (0.021) & (0.012) & (0.012)
\end{array}
$$

Length streak $=3$

$$
\begin{array}{cccccc}
\multicolumn{1}{c}{1} & \multicolumn{2}{c}{3} & \multicolumn{2}{c}{5} \\
0.035 & 0.051 & 0.094^{* *} & 0.100^{* * *} & -0.004 & -0.005 \\
(0.047) & (0.047) & (0.038) & (0.038) & (0.017) & (0.016)
\end{array}
$$

Length streak $=4$

$$
\begin{array}{cccccc}
\multicolumn{2}{c}{1} & \multicolumn{2}{c}{\frac{1}{2}} & \multicolumn{2}{c}{} \\
0.166^{* *} & 0.182^{* * *} & 0.169^{* *} & 0.153^{* *} & 0.022 & 0.013 \\
(0.065) & (0.064) & (0.070) & (0.065) & (0.029) & (0.028) \\
\hline
\end{array}
$$

\begin{tabular}{lcccccc}
\hline Number of participants & 75 & 75 & 63 & 63 & 72 & 72 \\
Subject FE & Y & N & Y & N & Y & N \\
Period FE & Y & Y & Y & Y & Y & Y \\
Number FE & Y & Y & Y & Y & Y & Y \\
Demographic controls & N & Y & N & Y & N & Y \\
Clustered st. err. & Y & Y & Y & Y & Y & Y \\
Obs & 30000 & 30000 & 25200 & 25200 & 28800 & 28800 \\
\hline
\end{tabular}

Notes: Cluster robust standard errors are provided in parentheses. Significance Coding: ${ }^{* * *}$ $1 \%, * *-5 \%, *-10 \%$. 
So far, our analysis has revealed a strong tendency in aggregate betting behavior to avoid past winning numbers in $T=1$, which then disappears in $T=2$ and $T=3$. To demonstrate that this pattern is the result of an increased inclination to bet on past winning numbers in $T=2$ and $T=3$, we next focus our analysis on individual-level data.

As a benchmark behavior, we consider i.i.d. betting strategies that conceptualize the absence of any preference for or against previous winning number(s). We then assess whether or not observed behavior deviates from this benchmark behavior. Observe that, under any i.i.d. strategy, the total count of bets on a previous winning number (i.e. to continue an on-going streak), denoted by $x$, follows a binomial distribution $x \sim B(n=40, p=i / 10)$ in treatment $T=i$, with $i=1,2,3$. Figure 4 depicts these theoretically expected density functions. We then bin participants based on the number of times they bet on a previous winning number and plot Gaussian kernel density estimates of these bin counts next to the i.i.d. densities in Figure 4. When compared to $T=2$ and $T=3$, the inclination to follow streaks in $T=1$ is skewed to the left relative to i.i.d. strategies. At the same time, the right-hand tails of the densities for treatments $T=2$ and $T=3$ are wider than their i.i.d. expectations, indicating an increased tendency to bet on previous winning numbers.

Figure 4: The Gambler's and Hot-Hand Fallacies at the Individual Level

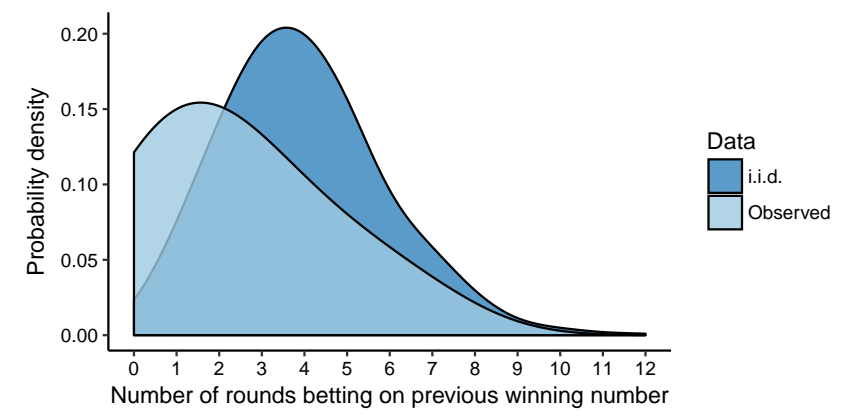

Figure 4a: Individual Behavior with $T=1$

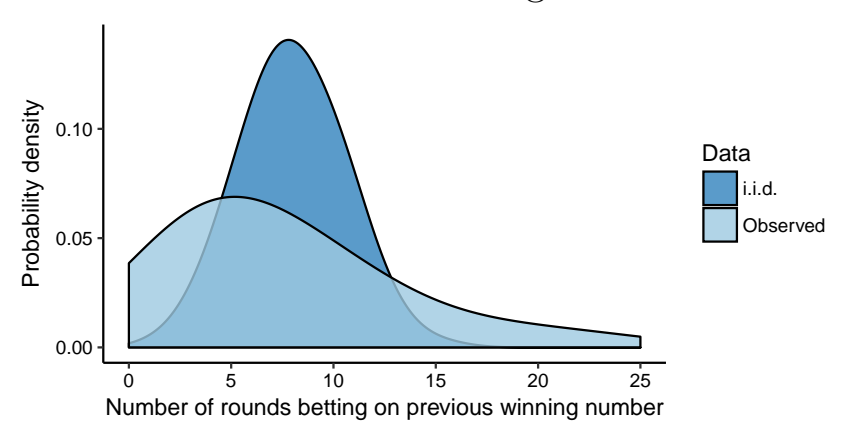

Figure 4b: Individual Behavior with $T=2$

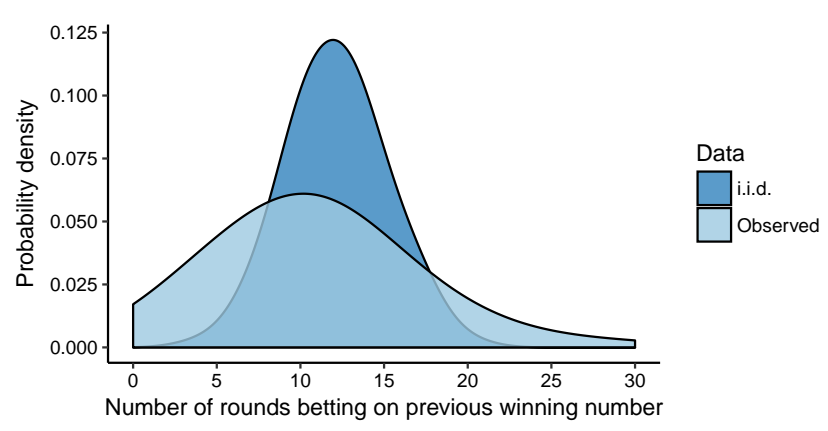

Figure 4c: Individual Behavior with $T=3$

We can now classify participants as seeking past numbers if they disproportionately continue streaks relative to the i.i.d. benchmark. Let $n_{j}$ denote the observed total count of bets on 
previous winning numbers by participant $j$. We consider two probability thresholds. Under the $10 \%$ probability threshold, participant $j$ is classified as a hot-hand player if $\operatorname{Pr}\left[x \geq n_{j}\right]<0.1$, i.e. if the probability of observing $n_{j}$ or more bets on previous winning numbers under an i.i.d. strategy is smaller than 10\%. Analogously, we consider a $5 \%$ probability threshold and classify $j$ as a hot-hand player if $\operatorname{Pr}\left[x \geq n_{j}\right]<0.05$. Under a threshold of $10 \%$, we observe $5.3 \%$, $20.6 \%$, and $16.7 \%$ hot-hand players in treatment $T=1, T=2$, and $T=3$, respectively. When measured at a threshold of $5 \%$, there are $0 \%, 17.5 \%$, and $12.5 \%$ hot-hand players in the three treatments, respectively. Table 7 reports the corresponding p-values of two-sided z-tests of proportions, using the correction procedure for family-wise error rates introduced in List et al. (2016). We find evidence that hot-hand behavior significantly increases from $T=1$ to $T=2$ and $T=3$, but no evidence for differences in hot-hand behavior between $T=2$ and $T=3.9$

Table 7: p-values of Two-Sided Z-Tests of Proportions

\begin{tabular}{|c|c|c|c|c|c|c|c|}
\hline \multicolumn{4}{|c|}{ Threshold at $10 \%$} & \multicolumn{4}{|c|}{ Threshold at 5\% } \\
\hline & VS & $T=2$ & $T=3$ & & vs & $T=2$ & $T=3$ \\
\hline$T=1$ & & 0.008 & 0.033 & $T=1$ & & 0.004 & 0.011 \\
\hline$T=2$ & & & 0.568 & $T=2$ & & & 0.433 \\
\hline
\end{tabular}

We conclude this subsection with an estimation of how likely it is that our novel empirical findings are recovering true associations. We hereby follow the formal framework developed in Maniadis et al. (2014). Specifically, we compute the Post-Study Probability (PSP) of a declared research finding. The PSP is the conditional probability that a significant finding is true. The probability itself depends on several parameters. We conventionally set the level significance at 0.05 and assume that we are the only research team that currently investigates our hypothesis. Furthermore, we calculate the post-hoc achieved power of our significant z-tests of proportions. The final parameter - the prior fraction of true scientific association examined in a research field - is unknown. We therefore consider various priors that represent meaningful values related to novel findings. Table 8 presents the resulting PSPs. For small and modest priors, our obtained PSPs are above 50\% (marked in bold). We therefore cautiously conclude that our main findings are likely to represent true associations.

\footnotetext{
${ }^{9}$ As an additional robustness check, we ran one-sided, pairwise exact Fisher-Boschloo tests. Even after accounting for family-wise error rates via Holm-Bonferroni, our conclusions from the main text remain unaffected. All p-values for comparisons of $T=2$ vs $T=3$ are below 0.017 . All p-values for comparisons of $T=2$ vs $T=3$ are above 0.746 .
} 
Table 8: Post-Study Probabilities (PSPs) with Different Priors

\begin{tabular}{ccccc}
\hline & \multicolumn{3}{c}{ Post-hoc power levels } \\
\cline { 2 - 2 } 0.95 & & 0.81 & 0.65 \\
\hline 0.01 & 0.16 & & 0.14 & 0.07 \\
0.05 & $\mathbf{0 . 5 0}$ & & 0.46 & 0.27 \\
0.15 & $\mathbf{0 . 7 7}$ & & $\mathbf{0 . 7 4}$ & $\mathbf{0 . 5 5}$ \\
0.25 & $\mathbf{0 . 8 6}$ & & $\mathbf{0 . 8 5}$ & $\mathbf{0 . 7 0}$ \\
0.35 & $\mathbf{0 . 9 1}$ & $\mathbf{0 . 9 0}$ & $\mathbf{0 . 7 9}$ \\
\hline
\end{tabular}

\subsection{Additional Analyses}

Why do participants exhibit more hot-hand behavior when we increase the number of balls drawn in a simulated lottery game? We present here a set of observations with regard to betting behavior that will motivate our answer to this question. For sake of brevity, we only state our main finding and refer interested readers to Appendix E for details.

First, we explore changes in behavior over the course of 40 rounds. We calculate the percentage of participants who bet on previous winners in each round over the course of the experiment, and then the percentage deviation from the treatment-specific base rates $(10 \%$, $20 \%$, and $30 \%$, respectively, for $T=1,2$, and 3 ). Figure 5 plots the adjusted deviation from the base rates, in which negative (positive) deviations indicate the gambler's fallacy (hot-hand fallacy). At the beginning of the experiment, deviations from the base rates are all negative, suggesting that participants in all treatments show a predisposition for the gambler's fallacy. This tendency persists in $T=1$, but not in $T=2$ or $T=3$. Participants in multiple-prize conditions alter their behavior as the game proceeds; evidence of a game-design induced change in beliefs about the underlying random process. This observation is corroborated by regression results on aggregate behavior restricting the sample to the first five rounds and last 35 rounds in the experiment. See Appendix $\mathrm{D}$ for details.

Second, we recorded each individual's decision time, i.e., the time it took a participant to enter his or her guess for the winning number. It is well established that decision time is a reliable indicator of task difficulty and task automaticity, with quicker decision time representing easier, more automatic decisions (Achtziger and Alós-Ferrer 2014, Alós-Ferrer et al. 2016). We ran panel regressions with individual random-effects to explore the intra-subject relationship between decision time and hot-hand behavior (continuing existing streaks); see detailed regression results in Appendix E. We find that hot-hand behavior is associated with slower decision times in $T=1$. This result suggests that following past winning numbers in $T=1$ is a more difficult decision than not betting on past numbers. In $T=2$ and $T=3$, however, 
Figure 5: Behavioral Change in Multiple-Prize Games

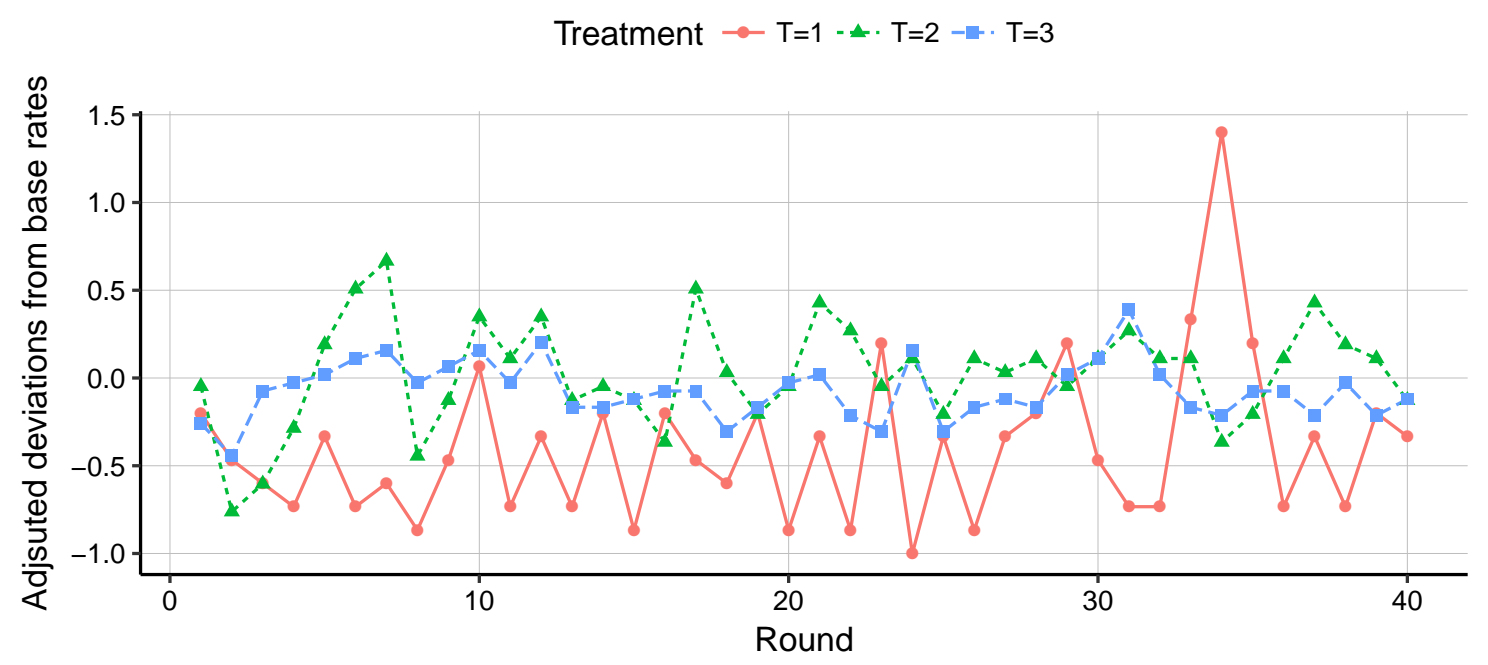

we observe a strong reversal in our results; betting on past winning numbers in these cases is a quicker decision than not betting on past winning numbers. These results suggest the existence of an underlying factor that triggers a change in participants' behavior and their perception of randomness when more balls are being drawn.

\subsection{Discussion}

We examined the role of game design - specifically, multiple outcomes in a random process - on the emergence of the gambler's and the hot-hand fallacies in a lottery game prediction task. Participants were asked to guess the outcome of simulated lottery games with one, two, or three winning numbers. Results show that, whereas the gambler's fallacy still prevailed in the single-prize game, it immediately disappeared when the number of outcomes was increased to two and three. At the individual level, we observed significantly more hot-hand behavior in multiple-prize games than in single-prize games. We infer from our observations that the lottery system design, in the form of the number of prizes drawn, systematically influenced participants' perceptions of randomness and, thereby, their betting behavior. These findings offer new insight into the prevalence of the gambler's and the hot-hand fallacies.

One plausible explanation for our observations is that different experiences across single and multiple-prize games can induce differences in beliefs about the random-generating process and, consequently, in betting behavior. Specifically, single and multiple-prize games differ with respect to how frequently past winning numbers re-occur. In our experiment, the theoretical probability of observing at least one repetition in two consecutive draws are, respectively, 10\%, $37.8 \%$, and $70.8 \%$, in $T=1, T=2$, and $T=3$ (realized relative frequencies are $10 \%, 32.5 \%$, and $70 \%$ ). For example, in round five of the experiment, participants observed no prior round 
with consecutive repetitions of winning numbers in $T=1$, but three and five prior rounds with consecutive winning numbers in $T=2$ and $T=3$, respectively. In our experimental data, gambler's fallacy effects emerge in the first few rounds across all treatments. This pattern suggests that all participants start out with a predisposition to avoiding past winning numbers, which is commonly associated with the incorrect belief in negative autocorrelation in i.i.d. processes. The subjective game experience in the single-prize game does not refute this belief as repetitions in winning numbers are rather rare events. However, participants in multipleprize games experience frequent repetitions in winning numbers, which challenges their prior belief. Eventually, they may abandon their initial belief in negative autocorrelation (gambler's fallacy), and some may adopt a new belief in positive autocorrelation (hot-hand fallacy), which is more consistent with their subjective game experience.

To lend further credence to our explanation, we conducted a small-scale replication study with 160 participants randomly assigned to the conditions $T=1$ and $T=3$. In replicating our main findings, we added to the design an incentivized belief-elicitation question about the unconditional probability of observing repetitions of winning numbers in two consecutive draws. We observe strong evidence that participants underestimate the probability of repetition in winning numbers in $T=3$. Specifically, participants state an average belief of $34.6 \%$, which falls short of both the theoretically correct probability $(70.8 \%)$ and the empirically realized one $(70 \%)$. These types of misjudgments in probabilities of repetition among multiple outcomes are common, as famously illustrated by the birthday paradox. Judged upon participants stated beliefs, the actual occurrence of consecutive winning numbers in $T=3$ is unexpected. In treatment $T=1$, participants overestimated the probability of repetition and stated an average belief of $24.8 \%$. However, the differences in stated beliefs between treatments are much smaller than theoretically expected and similar in magnitude.

We continue our discussion with some remarks on the validity of our experimental setup. First, our results with single-outcome games replicate/support some relevant findings in the literature. Based on Rabin's (2002) model, Asparouhova et al. (2009) predicted that the gambler's fallacy prevails when streaks are short, and the hot-hand fallacy emerges when streaks become longer. To the best of our knowledge, our experiment is the first to observe this pattern with a clear switching point from the gambler's fallacy to the hot-hand fallacy. Second, our experiment also replicates the empirical findings in Suetens et al. (2016), which are based on real Lotto data from Denmark. We applied the authors' approach to test for the presence of the gambler's fallacy. In line with their results, we find a reduction in bets on past winning numbers, which is consistent with the gambler's fallacy. They find a magnitude of between $1.6 \%$ and $3.8 \%$, depending on the sample under investigation, while we find a reduction of $4.3 \%$ in the treatment in which one ball is drawn. Finally, our findings are also consistent with observations by Wang et al. (2016), who found that participants avoid numbers that were 
drawn only once over the last six draws, while numbers that were drawn three or four times are relatively popular.

A potential confound is introduced by our "winner-take-all" payment scheme. Grand-prizes were awarded to the best-performing participant within each treatment, thereby inducing incentives to outperform others. In particular, sophisticated players could have adopted "antiimitation" strategies - that is, betting on numbers they believed others would not bet on. In this view, behavioral patterns cannot be attributed with certainty to the distortions in participants' perceptions of randomness, but could reflect their beliefs about other participants' behavior. The replication study we ran also contained a post-lottery-game incentivized recommendation task. Participants were asked to give strategic recommendations in the form of free-text to participants of future studies of the same game, earning them a small bonus payment each time their recommendation is found useful. Manually inspecting all 160 individual recommendations, we found none to explicitly or implicitly mention "to anti-imitate others" or something similar. We also found none to mention, in any form, our incentive scheme. We believe that these responses are sufficient evidence to conclude that an anti-imitate effect is not a concern with our data.

We conclude this section with a brief discussion on how our current experimental design came into place. For all experiments discussed below, participants had to guess one winning number for the next round and the best performing participant within a treatment was rewarded with a grand-prize. We first ran a batch of two similar experiments, one on Amazon's MTurk with 200 participants and a follow-up study with 47 course participants from one of our home universities. For the experiment on MTurk, a lottery machine randomly drew one or ten winning numbers from 0 to 99 (between subject design); we found no trace of the gambler's fallacy or the hot-hand fallacy in aggregate behavior. In the experiments with our students, one, five, or ten winning numbers were drawn from 10 to 59 (within subject design); we only found weak evidence for the hot-hand fallacy after long streaks in treatment $T=1$ and $T=5$, after playing treatment $T=10$. We concluded that the combination of the size of winning numbers drawn and the choice set (i.e., potential winning numbers) was sub-optimal for two reasons. First, we would have required a much bigger sample size for statistical inference with a large choice set (e.g., many numbers were never bet on, no reasonable baseline for betting frequencies on non-winning numbers could be established, no streaks with 1 out 100 balls drawn). Second, correct predictions were rather unlikely, thereby reducing participants' involvement in the game. In addition, we made important observations that guided our subsequent design choices. In particular, we observed a first indication of game experience (the order with which games were played) to influence betting behavior and a vast majority of our students reported never participating in real-life lottery games.

In response to these lessons learned, we came up with our final design as explained in this 
paper. We increased the level of realism in our presentation of the game and reduced the size of choice set to 10. This design offered enough flexibility to create different game experiences and provided higher leverage for statistical inference at both the aggregate and the individual level behavior. Finally, we run an unreported replication study with 150 participants on Prolific in which we always revealed winning numbers from the past five rounds on the screen (i.e. no history button). This design was motivated by Baron and Leider (2010) and all of our main results obtained. All data-sets are made publicly available on the authors' home-pages.

\section{Conclusion}

In this paper, we used data from two different lottery games to demonstrate the impact of game design in shaping two erroneous beliefs: the gambler's fallacy and the hot-hand fallacy. Field data showed that, when there are multiple prizes in a lottery game players tend to favor previous winning numbers, suggesting that they believe the same outcome will repeat in the future. We then ran a set of online experimental studies and found that behavior changed under different game designs; in particular, whereas a single-prize game leads to the gambler's fallacy - consistent with the current literature - multiple-prize games lead to more hot-hand behavior.

The notion that humans can be manipulated to believe in the hot-hand fallacy through appropriate game design has important ramifications in various fields. First, it provides a behavioral explanation for the "medium-prizes puzzle" (Haruvy et al. 2001) - that is, for why lottery-game operators typically offer prize distributions of a few large prizes and a large number of medium ones. This is surprising, especially if we assume that gamblers are typically riskseeking. There are two common explanations for this observation. One relies on prospect theory: A large number of medium prizes reduces the probability of losing from near certainty to some smaller probability. The other explanation follows the line of adaptive learning, that is: Human behavior is best captured by simple adaptive learning models, and actions that did well in the past tend to be adopted more frequently than those that did not. Thus, the presence of medium prizes slows down the agent's inclination to gamble less. Our paper provides another explanation: A large number of medium prizes can induce more players to believe in the hothand fallacy. This conviction reduces players' inclination to quit the game, and increases their desire to bet on those numbers they believe to have a higher probability of winning.

Second, our finding provides guidelines for designing lottery games that induce desirable behavior, which has important practical implications. A recent trend is for governments to encourage good civic behavior through the use of lottery games, the merits of which has been documented by Richard Thaler ${ }^{10}$. For instance, New Taipei City in Taiwan recently initiated

\footnotetext{
${ }^{10}$ See "Making Good Citizenship Fun," New York Times, February 13, 2012.
} 
a lottery as an inducement for dog owners (and other citizens) to clean up after their pets, with a grand prize of gold ingots worth as much as $\$ 2,000$. The Singapore government is also experimenting with this approach, and has implemented an incentive scheme in which commuters earn credit for each journey taken (with triple credit for off-peak journeys) toward a chance to win cash prizes in weekly lotteries. Our study highlights two features that render lottery games more effective for influencing behavior: (1) including a sufficiently large number of medium prizes (to induce more hot-hand believers), and (2) adding some mechanism that allows players to bet on numbers they believe to have a higher probability of winning(thereby increasing their incentive to participate in lottery games). One way to achieve the latter is to use personalized numbers (instead of random numbers) that players can easily relate to. The Dutch government uses this principle very effectively, by basing one of its state lotteries on postal codes. The idea is to make use of the near-miss effect, and also to exploit the lucky-store effect that has been shown to exist in various lottery games. The chances of winning may be falsely believed to be higher if the postal code has been drawn before (or is a near miss) in the previous draws, leading to greater participation.

\section{References}

Achtziger, A., and Alós-Ferrer, C. 2014. Fast or Rational? A Response-Times Study of Bayesian Updating, Management Science, 60(4), 923-938.

Alós-Ferrer, C., Granić, D.G., Kern, J., Wagner, A.K. 2016. Preference reversals: Time and again, Journal of Risk and Uncertainty, 52, 65-97.

Al-Ubaydli, O., and List, J.A. 2015. On the Generalizability of Experimental Results in Economics Handbook of Experimental Economic Methodology, Oxford University Press, New York, 2015.

Asparouhva, E., Hertzel, M., and Lemmon, M. 2009. Inference from streaks in random outcomes: Experimental evidence on beliefs in regime shifting and the law of small numbers, Management Science, 55(11), 1766-1782.

Ayton, P. and Fisher, I. 2004. The Gambler's Fallacy and the Hot-Handed Fallacy: Two faces of subjective randomness, Memory $\&$ Cognition, 32(8), 1369-1378.

Baron, G., and Leider, S. 2010. The role of experience in Gambler's Fallacy, Journal of Behavioral Decision Making, ,23(1), 117-129.

Box, G.E.P., and Jenkins, G.M. 1976. Time Series Analysis: Forecasting and Control, Holden-Day.

Boynton, D.M. 2003. Superstitious responding and frequency matching in the positive bias and gambler's fallacy effects, Organizational Behaviour and Human Decision Processes, 91, 119-127.

Burns, B.D. and Corpus, B. 2004. Randomness and inductions from steaks: "Gambler's fallacy" versus "hot-hand", Psychonomic Bulletin and Review, 11(1), 179-184. 
Camerer, C.F. 1989. Does the basketball market believe in the hot-hand? Review, 79(5), 1257-1261.

Caruso, E.M., Waytz, M., and Epley, N. 2010. The intentional mind and the hot-hand: Perceiving intentions makes streaks seem likely to continue, Cognition, 116(1), 149-153.

Chen, D.L., Moskowitz, T.J., and Shue, K. 2016. Decision-making under the gambler's fallacy: Evidence from asylum judges, loan officers, and baseball umpires, The Quarterly Journal of Economics, 131(3), 1181-1241.

Clark, L., Lawrence, A.J., Astley-Jones F., and Gray, N. 2009. Gambling near-misses enhance motivation to gamble and recruit win-related brain circuitry, Neuron , 61(3), 481-490.

Clotfelter, C., and Cook, D. 1993. The "Gambler's Fallacy' in lottery play, Management Science, 39(2), 1521-1525.

Croson, R. and Sundali, J. 2005. The gambler's fallacy and the hot-hand: Empirical data from casinos, Journal of Risk and Uncertainty, 30, 195-209.

Falk, R., and Konold, C. 1997 Making sense of randomness: Implicit encoding as a basis for judgment, Psychological Review, 104, 301-318.

Gilovich, T., Allone, R., and Tversky, A. 1985. The hot-hand in basketball: On the misperception of random sequences, Cognitive Psychology 17, 295-314.

Green, C.S., Benson, C., Kersten, D., and Schrater, P. 2010. Alterations in choice behavior by manipulations of world model, Proceedings of National Academy of Sciences .

Green, B., and Zwiebel, J. 2017. The Hot-Hand Fallacy: Cognitive Mistakes or Equilibrium Adjustments? Evidence from Major League Baseball, Management Science, forthcoming.

Guryan, J., and Kearney, M.S. 2008. Gambling at Lucky Stores: Empirical Evidence from State Lottery Sales, American Economic Review, 98(1), 458-473.

Hake, H.W. and Hyman, R. 1953. Perception of the statistical structure of a random series of binary symbol, Journal of Experimental Psychology, 45(1), 64-74.

Harrison, G.W., and List, J.A. 2004. Field Experiments, Journal of Economic Literature. 42(4), 1009-1055.

Haruvy E., Erev, I., and Sonsino, D. (2001). The medium prizes paradox: Evidence from a simulated casino, Journal of Risk and Uncertainty 22(3), 251-261.

List, J.A., Shaikh, A.M., and Xu, Y. 2016. Multiple Hypothesis Testing in Experimental Economics, NBER Working Paper No. 21875.

Maniadis, Z., Tufano, F., and List, J.A. 2014. One Swallow Doesn't Make a Summer: New Evidence on Anchoring Effects, American Economic Review, 104(1), 277-290.

Militana, E., Wolfson, E., and Cleaveland, J. M. 2010. An effect of inter-trial duration on the gambler's fallacy choice bias, Behavioural Processes, 84(1), 455459.

Navarrete, G. and C. Santamaria. 2012. Adding possibilities can reduce the Gambler's Fallacy: A naïve-probability paradox. Journal of Cognitive Psychology, 24(3), 306-312. 
Oskarsson, A.T., Van Boven, L., McClelland, G.H., and Hastie, R. 2009. What's next? Judging sequences of binary events, Psychological Bulletin, 135(2), 262-285.

Peer, E., . Brandimarte, L., Samat S., Acquisti, A. 2017. Beyond the Turk: Alternative platforms for crowdsourcing behavioral research, Journal of Experimental Social Psychology /, 70, 153163.

Rabin, M. 2002. Inference by believers in the law of small numbers, Quarterly Journal of Economics, 117(3), $775-816$.

Rabin, M., and Vayanos, D. 2010. The gambler's and hot-hand fallacies: Theory and applications, Review of Economic Studies, 77(2), 730-778.

Reid, R.L. 1986. The psychology of the near miss, Journal of Gambling Studies, 2(1), 32-39.

Stevenson, W.J. 2012. Operations Management 11/e, Mc Graw Hill, 2012.

Suetens, S., Galbo-Jørgensen, C.B., and Tyran, J. R. 2016. Predicting lotto numbers: A natural experiment on the gambler's fallacy and the hot-hand fallacy, Journal of the European Economic Association, 14(3), 584-607.

Sun, Y., and Wang, H. 2010. Gamblers fallacy, hot hand belief, and the time of patterns, Judgment and Decision Making, 5, 124-132.

Sundali, J. and Croson, R. 2006. Biases in casino betting: The hot-hand and the gambler's fallacy, Judgement and Decision Making, 1(1), 1-12.

Terrell, Dek. 1994. A test of the Gambler's Fallacy-evidence from pari-mutuel games, Journal of Risk and Uncertainty, 8(3), 309-317.

Tversky, A. 1974. Assessing Uncertainty, Journal of the Royal Statistical Society. Series B , 36(2), 148-159.

Tversky, A. and Kahneman, D. 1971. Belief in the law of small numbers, Psychological Bulletin, 76(2), 105-110.

Tversky, A. and Kahneman, D. 1974. Judgment under uncertainty: heuristics and biases, Science, 185, 1124-1131.

Wang, T.V., Potter van Loon, R.J.D., Van de Assem, M.J., and Van Dolder, D. 2016. Number preferences in lotteries, Judgment and Decision Making, 11(3), 243259. 


\section{A Randomness Check of The Lottery Data}

We check whether the true data-generating processes of $3 \mathrm{D}$ and $4 \mathrm{D}$ are indeed random, i.e., whether there exists positive or negative autocorrelation in the sequence of winning numbers. To do so, we use the auto-correlation function defined in Box and Jenkins (1976): Given measurements $Y_{1}, Y_{2}, \cdots, Y_{n}$ at time $X_{1}, X_{2}, \cdots, X_{n}$, the lag $k$ autocorrelation function is defined as:

$$
r_{k}=\frac{\sum_{i=1}^{N-k}\left(Y_{i}-\bar{Y}\right)\left(Y_{i+k}-\bar{Y}\right)}{\sum_{i=1}^{N}\left(Y_{i}-\bar{Y}\right)^{2}}
$$

In 3D, our dataset contains 4,466 consecutive draws from May 8, 2005 to October 30, 2017. We calculated the autocorrelation among these winning numbers with lag 1 , following the formula presented above. The lag-one autocorrelation is 0.006 , showing that these numbers are not autocorrelated.

In the $4 \mathrm{D}$ game, since the 23 winning numbers are drawn with replacement, we can treat the draw of each number as a data-generating process. However, since the winning numbers related to the 10 starters and the 10 consolations are presented in increasing order, those wining numbers have a positive autocorrelation. For this reason, we only test a sub-dataset with the first three winning numbers across 156 draws during a 1-year period. The autocorrelation among those numbers is 0.0392, again showing no significant autocorrelation among the winning numbers. 


\section{B Empirical Evidence}

Table 9: Payout Rate Statistics of Winning Numbers in 3D Game

\begin{tabular}{ccccccc}
\hline Numbers Repeating in & Data Point & Mean & Stdev & 25th Percentile & Median & 75th Percentile \\
\hline All data & 4466 & 0.49 & 0.27 & 0.32 & 0.43 & 0.59 \\
1 week & 27 & 0.34 & 0.36 & 0.18 & 0.23 & 0.36 \\
1 to 2 weeks & 27 & 0.35 & 0.12 & 0.27 & 0.31 & 0.42 \\
2 to 3 weeks & 22 & 0.40 & 0.24 & 0.26 & 0.30 & 0.54 \\
3 to 4 weeks & 31 & 0.42 & 0.20 & 0.29 & 0.40 & 0.47 \\
4 to 5 weeks & 28 & 0.47 & 0.26 & 0.29 & 0.40 & 0.53 \\
5 to 6 weeks & 34 & 0.46 & 0.23 & 0.31 & 0.38 & 0.56 \\
6 to 7 weeks & 31 & 0.43 & 0.16 & 0.32 & 0.41 & 0.53 \\
7 to 8 weeks & 30 & 0.58 & 0.32 & 0.34 & 0.49 & 0.76 \\
8 to 9 weeks & 37 & 0.45 & 0.21 & 0.33 & 0.38 & 0.52 \\
9 to 10 weeks & 29 & 0.45 & 0.20 & 0.31 & 0.39 & 0.52 \\
More than 10 weeks & 3180 & 0.49 & 0.24 & 0.33 & 0.44 & 0.59 \\
No Past Win & 990 & 0.50 & 0.34 & 0.30 & 0.42 & 0.59 \\
\hline
\end{tabular}

Table 10: Matched sample t-tests on payout rates for early vs late repetitions in 3D Game

\begin{tabular}{ccccc}
\hline$x$ & sample size & t-stat. & $p$-value & $95 \%$ confidence interval \\
\hline 3 & 70 & -5.07 & $3.20 \mathrm{e}-06$ & {$[-0.219,-0.095]$} \\
5 & 125 & -4.23 & $4.55 \mathrm{e}-05$ & {$[-0.147-0.053]$} \\
7 & 176 & -4.80 & $3.48 \mathrm{e}-06$ & {$[-0.120,-0.050]$} \\
9 & 231 & -4.13 & $5.08 \mathrm{e}-05$ & {$[-0.100,-0.035]$} \\
11 & 264 & -4.34 & $2.05 \mathrm{e}-05$ & {$[-0.094,-0.035]$} \\
\hline
\end{tabular}




\section{Experiment Design}

\section{General introduction to the study for all experimental conditions}

Thank you for your interest in this study. In this study, you are invited to play a simulated lottery game and your task is to guess which numbers a lottery-computer will draw. On top of your fixed earnings of 0.80 , you can earn a bonus payment of 10 for this study which will be awarded to every 50th respondent. More details are provided during the study. Please read all questions carefully and follow the on-screen instructions. Completing the survey will take about 9 minutes. If you close your browser before completing the survey, your data will be lost and you will have to start anew. Make sure that javascript is enabled in your browser. The survey works best on desktops and tablets, but might be inconvenient to complete on smartphones. Participant IDs and completion codes are automatically transferred. You can open this study in a new window.

\section{Introduction to the simulated lottery game with $T=1$}

Welcome to the Simulated Lottery Game! Today, you are invited to play a simulated lottery game. There will be 40 rounds in total. In each round, a lottery computer will randomly draw 1 numbered ball (0 - 9) from an urn. Each ball in the urn has an equal chance of being drawn. Your task is to guess, at the beginning of each round, which number will be drawn by the computer. If you guess it correctly, you earn 1 point. Roughly 70 respondents will play this game and the one with the highest points score will earn a bonus payment of 15 . It is therefore important that you read on-screen instructions carefully. Please click on NEXT to proceed. The computer will randomly draw 1 ball from an urn of 10 balls. The balls are numbered consecutively from 0 to 9 . That is, each number is represented by exactly one ball (one ball with 0 , one ball with $1, \ldots$, one ball with 9 ). Guess one number from 0 to 9 . If your number matches the number drawn, you earn 1 point. This game is repeated for 40 rounds.

\section{Introduction to the simulated lottery game with $T=2$}

Welcome to the Simulated Lottery Game! Today, you are invited to play a simulated lottery game. There will be 40 rounds in total. In each round, a lottery computer will randomly draw 2 numbered balls (0 - 9) from an urn. Each ball in the urn has an equal chance of being drawn. Your task is to guess, at the beginning of each round, which number will be drawn by the computer. If you guess it correctly, you earn 1 point. Roughly 70 respondents will play this game and the one with the highest points score will earn a bonus payment of 15 . It is therefore important that you read on-screen instructions carefully. Please click on NEXT to proceed. The computer will randomly draw 2 balls from an urn of 10 balls. The balls are numbered consecutively from 0 to 9 . That is, each number is represented by exactly one ball (one ball with 0 , one ball with $1, \ldots$, one ball with 9 ). Guess one number from 0 to 9 . If your number matches one of the numbers drawn, you earn 1 point. This game is repeated for 40 rounds.

Introduction to the simulated lottery game with $T=3$ 
Welcome to the Simulated Lottery Game! Today, you are invited to play a simulated lottery game. There will be 40 rounds in total. In each round, a lottery computer will randomly draw 3 numbered balls (0 - 9) from an urn. Each ball in the urn has an equal chance of being drawn. Your task is to guess, at the beginning of each round, which number will be drawn by the computer. If you guess it correctly, you earn 1 point. Roughly 70 respondents will play this game and the one with the highest points score will earn a bonus payment of 15 . It is therefore important that you read on-screen instructions carefully. Please click on NEXT to proceed. The computer will randomly draw 3 balls from an urn of 10 balls. The balls are numbered consecutively from 0 to 9 . That is, each number is represented by exactly one ball (one ball with 0 , one ball with $1, \ldots$, one ball with 9 ). Guess one number from 0 to 9 . If your number matches one of the numbers drawn, you earn 1 point. This game is repeated for 40 rounds. Figure 6 shows two screen shots from the experiment.

Figure 6: Screenshots from the experiment

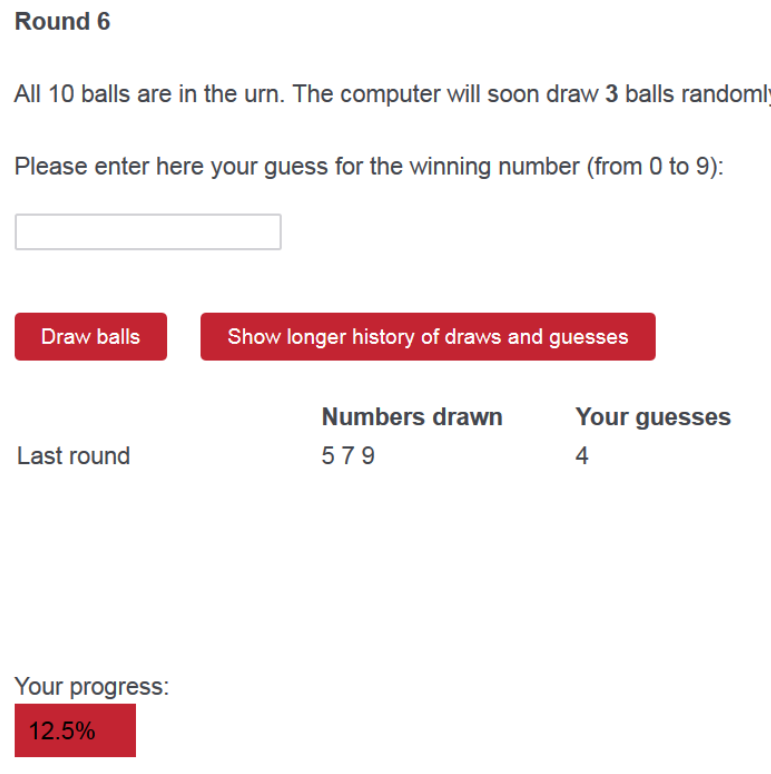

Figure 6a: Treatment $T=1$

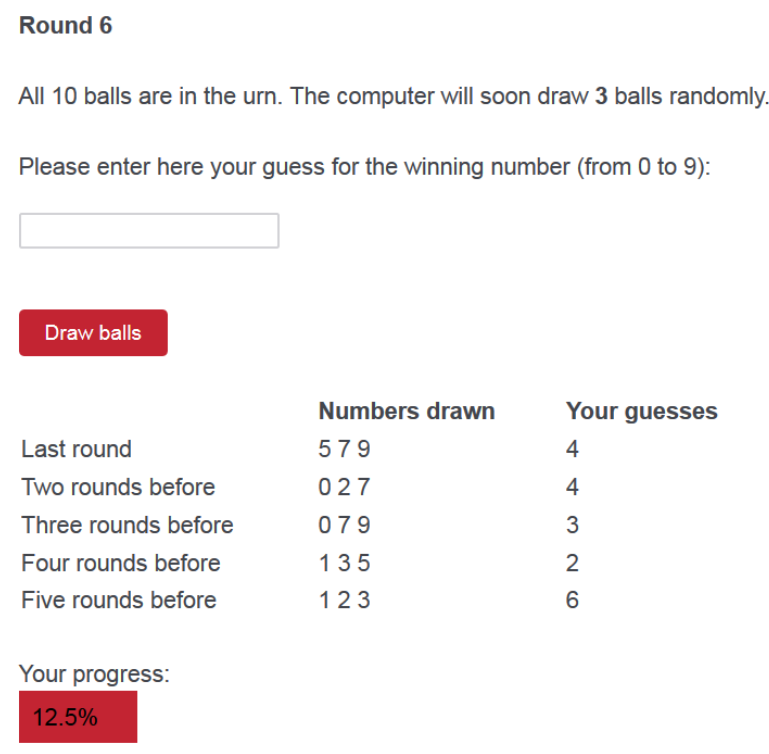

Figure 6b: Treatment $T=3$ 


\section{Regression Results Experiment Conditioning on the First 5 Rounds and the Last 35 Rounds}

Table 11: Average Marginal Effects of Probit Regression on Streak Length (Aggregate Level)

- First 5 Rounds

\begin{tabular}{lcccccc}
\hline & \multicolumn{2}{c}{$\mathrm{T}=1$} & \multicolumn{2}{c}{$\mathrm{T}=2$} & \multicolumn{2}{c}{$\mathrm{T}=3$} \\
\hline Length streak=1 & $-0.050^{* * *}$ & $-0.056^{* * *}$ & $-0.037^{* *}$ & $-0.034^{* *}$ & $-0.031^{* *}$ & $-0.032^{* *}$ \\
& $(0.000)$ & $(0.000)$ & $(0.012)$ & $(0.021)$ & $(0.013)$ & $(0.012)$ \\
Length streak=2 & & & -0.003 & 0.001 & -0.007 & -0.007 \\
& & & $(0.944)$ & $(0.987)$ & $(0.684)$ & $(0.692)$ \\
Length streak=3 & & & & & -0.021 & -0.021 \\
& & & & & $(0.572)$ & $(0.563)$ \\
\hline Number of participants & 75 & 75 & 63 & 63 & 72 & 72 \\
Subject FE & $\mathrm{Y}$ & $\mathrm{N}$ & $\mathrm{Y}$ & $\mathrm{N}$ & $\mathrm{Y}$ & $\mathrm{N}$ \\
Period FE & $\mathrm{Y}$ & $\mathrm{Y}$ & $\mathrm{Y}$ & $\mathrm{Y}$ & $\mathrm{Y}$ & $\mathrm{Y}$ \\
Number FE & $\mathrm{Y}$ & $\mathrm{Y}$ & $\mathrm{Y}$ & $\mathrm{Y}$ & $\mathrm{Y}$ & $\mathrm{Y}$ \\
Demographic controls & $\mathrm{N}$ & $\mathrm{Y}$ & $\mathrm{N}$ & $\mathrm{Y}$ & $\mathrm{N}$ & $\mathrm{Y}$ \\
Clustered std. err. & $\mathrm{Y}$ & $\mathrm{Y}$ & $\mathrm{Y}$ & $\mathrm{Y}$ & $\mathrm{Y}$ & $\mathrm{Y}$ \\
Obs & 3750 & 3750 & 3150 & 3150 & 3600 & 3600 \\
\hline
\end{tabular}

Notes: Cluster robust standard errors are provided in parentheses. Significance Coding: *** - $1 \%$, $* *-5 \%, *-10 \%$. 
Table 12: Average Marginal Effects of Probit Regression on Streak Length (Aggregate Level)

- Last 35 Rounds

\begin{tabular}{lcccccc}
\hline & \multicolumn{2}{c}{$\mathrm{T}=1$} & \multicolumn{2}{c}{$\mathrm{T}=2$} & \multicolumn{2}{c}{$\mathrm{T}=3$} \\
\hline Length streak=1 & $-0.051^{* * *}$ & $-0.052^{* * *}$ & 0.001 & 0.006 & -0.012 & -0.009 \\
& $(0.000)$ & $(0.000)$ & $(0.925)$ & $(0.671)$ & $(0.214)$ & $(0.320)$ \\
Length streak=2 & $-0.053^{* * *}$ & $-0.044^{* *}$ & 0.015 & 0.021 & -0.001 & 0.001 \\
& $(0.004)$ & $(0.021)$ & $(0.459)$ & $(0.329)$ & $(0.969)$ & $(0.928)$ \\
Length streak=3 & 0.034 & 0.051 & $0.094^{* *}$ & $0.101^{* * *}$ & -0.000 & -0.001 \\
& $(0.462)$ & $(0.274)$ & $(0.012)$ & $(0.009)$ & $(0.995)$ & $(0.978)$ \\
Length streak=4 & $0.166^{* *}$ & $0.182^{* * *}$ & $0.168^{* *}$ & $0.152^{* *}$ & 0.022 & 0.013 \\
& $(0.011)$ & $(0.004)$ & $(0.016)$ & $(0.017)$ & $(0.453)$ & $(0.640)$ \\
\hline Number of participants & 75 & 75 & 63 & 63 & 72 & 72 \\
Subject FE & $\mathrm{Y}$ & $\mathrm{N}$ & $\mathrm{Y}$ & $\mathrm{N}$ & $\mathrm{Y}$ & $\mathrm{N}$ \\
Period FE & $\mathrm{Y}$ & $\mathrm{Y}$ & $\mathrm{Y}$ & $\mathrm{Y}$ & $\mathrm{Y}$ & $\mathrm{Y}$ \\
Number FE & $\mathrm{Y}$ & $\mathrm{Y}$ & $\mathrm{Y}$ & $\mathrm{Y}$ & $\mathrm{Y}$ & $\mathrm{Y}$ \\
Demographic controls & $\mathrm{N}$ & $\mathrm{Y}$ & $\mathrm{N}$ & $\mathrm{Y}$ & $\mathrm{N}$ & $\mathrm{Y}$ \\
Clustering & $\mathrm{Y}$ & $\mathrm{Y}$ & $\mathrm{Y}$ & $\mathrm{Y}$ & $\mathrm{Y}$ & $\mathrm{Y}$ \\
Obs & 26250 & 26250 & 22050 & 22050 & 25200 & 25200 \\
\hline
\end{tabular}

Notes: Cluster robust standard errors are provided in parentheses. Significance Coding: ${ }^{* *}$ $1 \%, * *-5 \%, *-10 \%$. 


\section{E Regression Results on Decision Time}

We followed the empirical strategy in Achtziger and Alós-Ferrer (2014) and Alós-Ferrer et al. (2016) and adopted a GLS panel approach with random effects at the individual level to analyze the impact of various variables on decision times. Our dependent variable in all models is the logarithm of Decision Time measured from the onset of presentation of the decision screen until submission of a guess for a winning number. Independent observations are taken at the individual-guess-period level. The estimation results are reported in Table 13 . We include dummies for our multiple-prize treatments $T=2$ and $T=3$ and a dummy for whether the guess of an individual continues an ongoing streak (Hot Hand). As reported in the main text, hothand behavior was slower than non-hot-hand behavior in $T=1$, whereas hot-hand behavior was quicker in than non-hot-hand behavior in multiple-prize games. We include additional control variables to validate decision times and to control for possible confounding factors. Participants guessed faster in later periods of the experiment, evidence of learning and taskexperience effects. Decisions were slower when the history button was clicked. Naturally, it is time consuming to click the button and process the resulting additional information displayed. We also observe that older participants were slower than younger ones, and that women and participants associating themselves to as Trans gender were slower than men. The lotto playing habits of participants had no influence on the speed of decisions. 
Table 13: GLS panel regression with random effects at the individual level and cluster robust standard errors (reported in parentheses). Significance coding: *** $-1 \%, * *-5 \%, *-10 \%$.

\begin{tabular}{|c|c|c|c|}
\hline $\begin{array}{l}\text { Dependent Var } \\
\text { Log(Decision Time) }\end{array}$ & Model (1) & Model (2) & Model (3) \\
\hline Hot Hand & $\begin{array}{c}0.127^{* * *} \\
(0.048)\end{array}$ & $\begin{array}{c}0.083^{*} \\
(0.043)\end{array}$ & $\begin{array}{l}0.083^{*} \\
(0.043)\end{array}$ \\
\hline $\mathrm{T}=2$ & $\begin{array}{c}0.160^{* * *} \\
(0.062)\end{array}$ & $\begin{array}{c}0.091 \\
(0.061)\end{array}$ & $\begin{array}{c}0.069 \\
(0.056)\end{array}$ \\
\hline $\mathrm{T}=3$ & $\begin{array}{c}0.061 \\
(0.068)\end{array}$ & $\begin{array}{l}-0.001 \\
(0.060)\end{array}$ & $\begin{array}{l}-0.018 \\
(0.059)\end{array}$ \\
\hline Hot Hand $\times \mathrm{T}=2$ & $\begin{array}{l}-0.201^{* * *} \\
(0.058)\end{array}$ & $\begin{array}{l}-0.183^{* * *} \\
(0.050)\end{array}$ & $\begin{array}{c}-0.181^{* * *} \\
(0.050)\end{array}$ \\
\hline Hot Hand $\times \mathrm{T}=3$ & $\begin{array}{c}-0.162^{* * *} \\
(0.052)\end{array}$ & $\begin{array}{c}-0.125^{* * *} \\
(0.047)\end{array}$ & $\begin{array}{c}-0.125^{* * *} \\
(0.047)\end{array}$ \\
\hline Period & $\begin{array}{c}-0.017^{* * *} \\
(0.001)\end{array}$ & $\begin{array}{l}-0.014^{* * *} \\
(0.001)\end{array}$ & $\begin{array}{c}-0.014^{* * *} \\
(0.001)\end{array}$ \\
\hline Hotness Number & & $\begin{array}{c}-0.602^{* * *} \\
(0.044)\end{array}$ & $\begin{array}{c}-0.595^{* * *} \\
(0.044)\end{array}$ \\
\hline Click History Button & & $\begin{array}{c}1.073^{* * *} \\
(0.052)\end{array}$ & $\begin{array}{l}1.075^{* * *} \\
(0.052)\end{array}$ \\
\hline Age & & & $\begin{array}{c}0.012^{* * *} \\
(0.002)\end{array}$ \\
\hline Female & & & $\begin{array}{l}0.101^{* *} \\
(0.048)\end{array}$ \\
\hline Trans* & & & $\begin{array}{c}0.527^{* * *} \\
(0.094)\end{array}$ \\
\hline Playing Lotto Monthly & & & $\begin{array}{c}0.095 \\
(0.070)\end{array}$ \\
\hline Playing Lotto < Monthly & & & $\begin{array}{c}0.022 \\
(0.060)\end{array}$ \\
\hline Playing Lotto Never & & & $\begin{array}{l}-0.027 \\
(0.062)\end{array}$ \\
\hline Income & & & $\begin{array}{l}-0.009 \\
(0.010)\end{array}$ \\
\hline Constant & $\begin{array}{l}1.579^{* * *} \\
(0.052) \\
\end{array}$ & $\begin{array}{c}1.613^{* * *} \\
(0.047) \\
\end{array}$ & $\begin{array}{c}1.164^{* * *} \\
(0.104)\end{array}$ \\
\hline Number of participants & 210 & 210 & 210 \\
\hline Obs & 8400 & 8400 & 8400 \\
\hline Clustered std. err. & $\mathrm{Y}$ & $\mathrm{Y}$ & $\mathrm{Y}$ \\
\hline Overall $\mathrm{R}^{2}$ & 0.098 & 0.264 & 0.313 \\
\hline Wald $\chi^{2} \mathrm{p}$ & 0.000 & 0.000 & 0.000 \\
\hline
\end{tabular}

Notes: Cluster robust standard errors are provided in parentheses. Significance Coding: $* * *-1 \%, * *-5 \%, *-10 \%$. 